\title{
Hybrid quantum-classical modeling of quantum dot devices
}

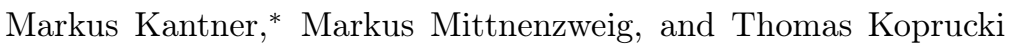 \\ Weierstrass Institute for Applied Analysis and Stochastics, \\ Mohrenstr. 39, 10117 Berlin, Germany
}

\begin{abstract}
The design of electrically driven quantum dot devices for quantum optical applications asks for modeling approaches combining classical device physics with quantum mechanics. We connect the well-established fields of semi-classical semiconductor transport theory and the theory of open quantum systems to meet this requirement. By coupling the van Roosbroeck system with a quantum master equation in Lindblad form, we introduce a new hybrid quantum-classical modeling approach, which provides a comprehensive description of quantum dot devices on multiple scales: It enables the calculation of quantum optical figures of merit and the spatially resolved simulation of the current flow in realistic semiconductor device geometries in a unified way. We construct the interface between both theories in such a way, that the resulting hybrid system obeys the fundamental axioms of (non-)equilibrium thermodynamics. We show that our approach guarantees the conservation of charge, consistency with the thermodynamic equilibrium and the second law of thermodynamics. The feasibility of the approach is demonstrated by numerical simulations of an electrically driven single-photon source based on a single quantum dot in the stationary and transient operation regime.
\end{abstract}

PACS numbers: 05.30.-d, 42.50.-p, 73.63.Kv, 85.30.De, 85.35.-p, 85.60.Bt

Keywords: device simulation, quantum dots, Lindblad equation, quantum-classical coupling, single-photon sources

\section{INTRODUCTION}

Semiconductor quantum dots (QDs) are zerodimensional nanostructures which provide a discrete spectrum of electronic states due to the confinement of charge carriers in all spatial dimensions. Because of their tunable electro-optical properties and their easy integration into dielectric microcavities, QDs have attracted considerable attention in particular for applications in solid-state based optoelectronic devices [1/5]. These include e.g. highly efficient semiconductor micro- and nanolasers with a few or even a single QD as gain medium [6 10], semiconductor optical amplifiers [1], and quantum light sources such as single-photon emitters and sources of entangled photon pairs [12 15]. Applications comprise optical communication and quantum information processing [13, 14, 16, quantum cryptography [17, optical computing [18] and bio-chemical sensing [19].

Currently, quantum optics is making the leap from the lab to commercial applications. On this way, device engineers will need simulation tools, which combine classical device physics with models from quantum mechanics. The modeling and simulation of electrically driven semiconductor devices containing QDs constitutes a considerable challenge. On the one hand, modern optoelectronic devices increasingly employ quantum optical effects based on coherent light matter interaction, entanglement, photon counting statistics and non-classical correlations, which require a quantum mechanical description of the charge carriers and the optical field. In the last decades, light emitting devices based on a single or a few QDs have been successfully described by quantum mas-

\footnotetext{
* kantner@wias-berlin.de
}

ter equations (QMEs) for the density matrix [7, 9, 20, which enable a detailed description of the dynamics of open quantum systems. On the other hand, the simulation of electrically driven devices requires a spatially resolved description of the current injection from the highly doped barriers and metal contacts into the optically active region containing the semiconductor QDs. The carrier transport problem is well described by semi-classical transport models such as the van Roosbroeck system [21], which describes the drift and diffusion of carriers within their self-consistently generated electric field. The van Roosbroeck system has been applied previously to QD devices, in particular to QD-based intermediate band solar cells [22, 23] and for the optimization of the current injection in single-photon sources 24].

Both fields, the theory of open quantum systems and the semi-classical semiconductor transport theory, are well developed and established for several decades. The scope of this paper is the self-consistent coupling of both theories in order to obtain a comprehensive description of QD-based optoelectronic devices on multiple scales. Therefore, the interface connecting both systems will be constructed in such a way, that the resulting hybrid quantum-classical model guarantees the conservation of charge, consistency with the thermodynamic equilibrium and the second law of thermodynamics.

The paper is organized as follows: In Sec. III the model equations are introduced and the physical properties of the hybrid quantum-classical model are discussed. We present the structure of the coupling terms between both systems and investigate important features such as the conservation of charge. In Sec. III the consistency of the model equations with fundamental axioms of (non-)equilibrium thermodynamics is investigated. In particular, we construct the thermodynamic equilibrium solution by minimizing the grand potential of the coupled 
system and show that the hybrid model obeys the second law of thermodynamics. In Sec. IV the approach is applied to the simulation of an electrically driven singlephoton source based on a single QD. We study the stationary and transient excitation regime by numerical simulations and show how the model allows to compute the decisive quantum optical figures of merit along with the spatially resolved carrier transport characteristics. Finally, in Sec. $\mathrm{V}$ we give an outlook on extensions of the approach.

\section{MODEL EQUATIONS}

We consider a hybrid quantum-classical model that self-consistently couples semi-classical transport theory to a kinetic equation for the quantum mechanical density matrix. The latter one is a QME in a Born-Markov and secular (rotating wave) approximation that describes the evolution of an open quantum system which interacts with its macroscopic environment [25]28]. In the following, the open quantum system is given by a single or a few QDs. Our approach is based on the assumption that the charge carriers can be separated into (free) continuum carriers and (bound) carriers confined to QDs, which is typically met for optoelectronic devices operating close to flat band conditions (weak electric fields) 29] 31. The model equations read

$$
\begin{aligned}
-\nabla \cdot \varepsilon \nabla \psi & =q(p-n+C+Q(\rho)), \\
\partial_{t} n-\frac{1}{q} \nabla \cdot \mathbf{j}_{n} & =-R-S_{n}(\rho ; n, p, \psi), \\
\partial_{t} p+\frac{1}{q} \nabla \cdot \mathbf{j}_{p} & =-R-S_{p}(\rho ; n, p, \psi), \\
\frac{\mathrm{d}}{\mathrm{d} t}=\mathcal{L}(\rho ; n, p, \psi) & =-\frac{i}{\hbar}[H, \rho]+\mathcal{D}(\rho ; n, p, \psi)
\end{aligned}
$$

on the domain $\Omega \subset \mathbb{R}^{3}$. The system (1)-(4) is subject to initial conditions and boundary conditions modeling electrical contacts and other interfaces [32. See Appendix A for the boundary conditions considered throughout this paper. A schematic illustration of the modeling approach is shown in Fig. 1 .

The model (1)-(4) differs from the typical quantum optical setting by explicitly considering the spatially resolved semi-classical carrier transport equations (1)-(3) as a part of the system under investigation. As a consequence, here the notion "reservoir" is employed differently from the standard quantum optics literature. In the following, the term reservoir refers to the electrical contacts connected to the semiconductor device and the surrounding heat bath, which must be distinguished from the classical or macroscopic environment of the quantum system, see Fig. 2. The continuum carriers, which represent the electronic part of the classical environment of the quantum system, evolve according to the van Roosbroeck system.

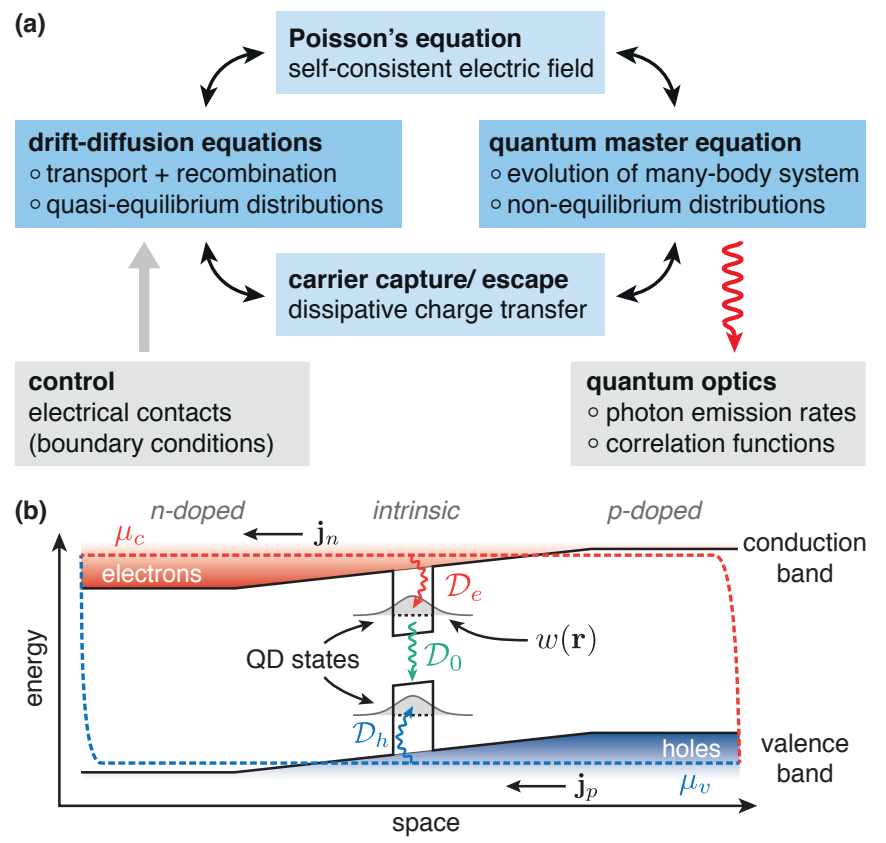

Figure 1. (a) Illustration of the hybrid quantum-classical modeling approach. A quantum system described by a QME is self-consistently coupled to the semi-classical transport equations for the freely roaming continuum carriers. Both (sub-)systems exchange charge by capture and escape of carriers and interact via their self-consistently generated electric field. (b) Schematic band diagram of the hybrid system in a $1 \mathrm{D}$ cross-section of a $\mathrm{p}-\mathrm{i}-\mathrm{n}$ diode with a single QD embedded in the intrinsic zone. The dissipative interactions of the quantum system with its classical environment are described by dissipation superoperators of Lindblad type $\mathcal{D}_{e}, \mathcal{D}_{h}$ and $\mathcal{D}_{0}$ for carrier capture and escape, recombination etc. The interaction domain is determined by the spatial profile $w$.

\section{A. Van Roosbroeck system}

Eqns. (1)-(3) represent the standard van Roosbroeck system, extended by additional terms that constitute the coupling to the quantum system. Poisson's Eq. (1) describes the electrostatic potential $\psi$ generated by the free electron and hole densities $n$ and $p$, the (stationary) built-in doping profile $C$ and the expectation value of the charge density $Q(\rho)$ of the carriers confined to the QDs. The dielectric permittivity of the semiconductor material is given by $\varepsilon=\varepsilon_{0} \varepsilon_{r}$ and $q$ denotes the elementary charge. The continuity equations (2)-(3) describe the flux of free electrons and holes in the presence of recombination and transitions between free and bound states. The (net-)recombination rate $R$ includes several recombination channels such as Shockley-Read-Hall recombination, spontaneous emission and Auger recombination. Moreover, carriers can be scattered from the continuum to the QDs which is described by the (net-)capture rates $S_{n}$ and $S_{p}$. The van Roosbroeck system must be augmented with additional state equations for the free car- 
rier densities

$$
\begin{aligned}
& n=N_{c} F_{1 / 2}\left(\beta\left(\mu_{c}-E_{c}+q \psi\right)\right), \\
& p=N_{v} F_{1 / 2}\left(\beta\left(E_{v}-q \psi-\mu_{v}\right)\right)
\end{aligned}
$$

and the electrical current densities

$$
\begin{aligned}
\mathbf{j}_{n} & =\frac{1}{q} \sigma_{n} \nabla \mu_{c}, \\
\mathbf{j}_{p} & =\frac{1}{q} \sigma_{p} \nabla \mu_{v} .
\end{aligned}
$$

Here, $N_{c}$ and $N_{v}$ denote the effective density of states of the conduction and valence band and $E_{c}$ and $E_{v}$ are the respective band edge energies. The inverse temperature $\beta=\left(k_{B} T\right)^{-1}$ is considered as a fixed parameter and

$$
F_{\nu}(\eta)=\frac{1}{\Gamma(\nu+1)} \int_{0}^{\infty} \mathrm{d} \xi \frac{\xi^{\nu}}{e^{\xi-\eta}+1}
$$

is the Fermi-Dirac integral of order $\nu$. The state equations (5) describe thermalized carrier ensembles in a quasi-equilibrium distribution, where the quasi-Fermi energies of the conduction band $\mu_{c}$ and the valence band $\mu_{v}$ parametrize the deviation from the thermodynamic equilibrium. In accordance with linear irreversible thermodynamics, the current densities are driven by the gradients of the quasi-Fermi energies [33]. The electrical conductivities $\sigma_{n}=q M_{n} n, \sigma_{p}=q M_{p} p$ are products of the carrier densities and the carrier mobilities $M_{n / p}$.

\section{B. Quantum master equation}

The state of the quantum system is described by the density matrix $\rho$, which is subject to the QME (4). Here, the quantum system represents a many-body problem describing the charge carriers confined to QDs and possibly further quasi-particles, e.g. cavity photons, phonons or exciton-polaritons (dressed states).

The Hamiltonian in Eq. (4) takes the form

$$
H=H_{0}+H_{I},
$$

where $H_{0}$ describes the single-particle energies of the confined electrons and holes (and possibly additional particle species). The interaction Hamiltonian $H_{I}$ is assumed to commute with the charge number operator of the quantum system

$$
N=n_{e}-n_{h}
$$

( $n_{e}$ and $n_{h}$ are the number operators of the bound electrons and holes) such that the Hamiltonian part of the evolution conserves the net charge

$$
[H, N]=0 .
$$

This imposes only a weak restriction on $H_{I}$ and allows e.g. for Coulomb interaction between the confined carriers (configuration interaction) as well as coherent lightmatter interaction.
We assume the quantum system to be embedded in a semiconductor device, which represents a macroscopic environment with an infinitely large number of degrees of freedom. The interactions of the quantum system with its environment, e.g. the exchange of energy and charge via recombination and capture or escape of carriers, represent dissipative processes that are described by the dissipation superoperator $\mathcal{D}$. Within the limit of weak system-reservoir coupling one obtains by using the Born-Markov and secular (rotating wave) approximation a dissipation superoperator in Lindblad form [28, 34]

$$
\begin{aligned}
\mathcal{D}(\rho ; \chi) & =\sum_{\alpha \in I_{\alpha}} \mathcal{D}_{\alpha}(\rho ; \chi) \\
& =\sum_{\alpha \in I_{\alpha}}\left(\gamma_{\alpha}(\chi) L_{A_{\alpha}}(\rho)+\hat{\gamma}_{\alpha}(\chi) L_{A_{\alpha}^{\dagger}}(\rho)\right)
\end{aligned}
$$

with the Lindblad superoperator

$$
L_{A}(\rho)=A \rho A^{\dagger}-\frac{1}{2}\left\{A^{\dagger} A, \rho\right\} .
$$

The admitted irreversible interactions between the quantum system and its environment are indexed by $\alpha \in I_{\alpha}$. The environment considered in this paper is a tensor product of multiple thermal states. This comprises a bosonic heat bath (lattice phonons, thermal radiation) and the thermalized carrier ensembles, which are subject to the van Roosbroeck system (1)-(3). In the hybrid model, the forward and backward transition rates $\gamma_{\alpha}$ and $\hat{\gamma}_{\alpha}$ depend on the state of the macroscopic environment, which is indicated here by the state vector $\chi$. Under the assumptions and approximations outlined above, the dissipation superoperator can be additively decomposed into various channels as given in Eq. (9) [34]. A QME in Lindblad form ensures the preservation of trace, hermiticity and (complete) positivity of the density matrix [26, 27]. The symbol $\{A, B\}=A B+B A$ denotes the anti-commutator. The operators $A_{\alpha}$ represent the quantum jump operators, which are projectors between different eigenstates of $H$. Following the standard construction of a Lindblad-QME for a weak system-reservoir interaction [28] (extended to the case of variable charge number here), we require the jump operators to satisfy

$$
\begin{aligned}
& {\left[H, A_{\alpha}\right]=-\hbar \omega_{\alpha} A_{\alpha},} \\
& {\left[N, A_{\alpha}\right]=-\ell_{\alpha} A_{\alpha},}
\end{aligned}
$$

where $\hbar \omega_{\alpha}$ denotes the transition energy and $\ell_{\alpha} \in \mathbb{Z}$ quantifies the charge transfer of the interaction described by $A_{\alpha}$. In order to classify the dissipation superoperators with respect to their effect on the charge of the quantum system, we collect the dissipators belonging to equal values of $\ell_{\alpha}$ and introduce the notation

$$
\mathcal{D}(\rho ; \chi)=\mathcal{D}_{e}(\rho ; \chi)+\mathcal{D}_{h}(\rho ; \chi)+\mathcal{D}_{0}(\rho ; \chi),
$$

where we have split the index set $I_{\alpha}$ into three disjoint subsets $I_{\alpha}=I_{e} \cup I_{h} \cup I_{0}$. With $\ell_{\alpha \in I_{e}}=-1$ and 
$\ell_{\alpha \in I_{h}}=+1$, the dissipators $\mathcal{D}_{e}$ and $\mathcal{D}_{h}$ can change the charge of the quantum system (by capture and escape of electrons and holes), whereas the processes described by $\mathcal{D}_{0}$ with $\ell_{\alpha \in I_{0}}=0$ leave the charge invariant (e.g. spontaneous emission, photon absorption, intraband carrier relaxation, outcoupling of cavity photons). Simultaneous capture of multiple carriers with $\left|\ell_{\alpha}\right| \geq 2$ is neglected here. From Eq. (10b) and $\ell_{\alpha \in I_{0}}=0$ one easily obtains

$$
\operatorname{tr}\left(N \mathcal{D}_{0}(\rho ; \chi)\right)=0 .
$$

Throughout this paper, we restrict ourselves to dissipation superoperators which satisfy the quantum detailed balance condition with respect to the thermodynamic equilibrium [35, 36]. This requires a certain relationship between the forward and backward transition rates $\gamma_{\alpha}$ and $\hat{\gamma}_{\alpha}$, which will be discussed in Sec. IIIC. In the case of degenerate energy spectra, the traditional secular approximation must be modified to properly account for degenerate eigenstate coherences. As shown in [37, this can be done in a thermodynamically consistent way. Finally, we remark that the Lamb-Shift is neglected throughout this paper.

\section{Macroscopic coupling terms and charge conservation}

By taking the time derivative of Poisson's Eq. (1) and using Eq. (2)-(3), we obtain the continuity equation

$$
\nabla \cdot \mathbf{j}_{\text {tot }}=q\left(\partial_{t} Q-S_{p}+S_{n}\right)
$$

for the total current density $\mathbf{j}_{\text {tot }}=\mathbf{j}_{n}+\mathbf{j}_{p}+\partial_{t} \mathbf{D}$. Besides the flux of charge carriers, it also includes the displacement current density $\partial_{t} \mathbf{D}=-\varepsilon \partial_{t} \nabla \psi$. For the sake of simplicity, we consider a quantum system comprising only a single QD. The generalization of the approach outlined below to the case of multiple QDs is straightforward. We approximate the electric charge density of the QD by the expectation value of the (net-)charge operator

$$
Q(\rho)=-w(\mathbf{r}) \operatorname{tr}(N \rho),
$$

where $w$ models the spatial profile of the captured carriers, which is assumed to be identical for all carriers. The function $w$ is normalized such that $\int_{\Omega} \mathrm{d}^{3} r w(\mathbf{r})=1$. The spatial profile $w$ replaces the absolute squares of the many-body wave functions of the bound carriers. The actual spatial distributions of the confined carriers differ only on a small length scale, which can be safely neglected in the simulation of macroscopic charge transport. In the form of Eq. (13), the model accounts for long range electrostatic correlations induced by the confined carriers.

Using Eqns. (4), (8), (11) and (12), the time derivative of Eq. (13) is obtained as

$$
\begin{aligned}
\partial_{t} Q= & -w(\mathbf{r}) \operatorname{tr}\left(N \mathcal{D}_{e}(\rho ; n, p, \psi)\right) \\
& -w(\mathbf{r}) \operatorname{tr}\left(N \mathcal{D}_{h}(\rho ; n, p, \psi)\right) .
\end{aligned}
$$
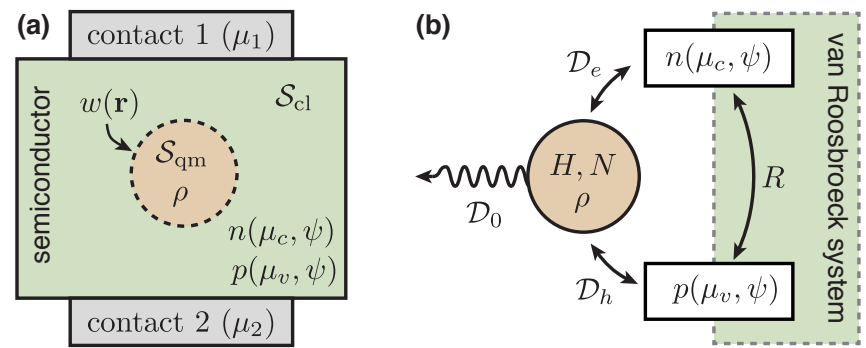

Figure 2. (a) Spatial arrangement of the system $\mathcal{S}$ and the electrical contacts (reservoirs). The system $\mathcal{S}$ consists of a classical subsystem $\mathcal{S}_{\mathrm{cl}}$ and a quantum mechanical subsystem $\mathcal{S}_{\mathrm{qm}}$ in the interior of the classical domain. The classical system is in contact with several electric contacts, which act as charge reservoirs and are characterized by their chemical potentials $\mu_{i}$ and a common inverse temperature $\beta$. The reservoirs enter the equations via Dirichlet boundary conditions. (b) Illustration of the coupling scheme. The quantum system is coupled to the van Roosbroeck system via charge transfer mediated by dissipation superoperators $\mathcal{D}_{e}$ and $\mathcal{D}_{h}$. Further decay processes, which keep the charge of the quantum system invariant, are described by $\mathcal{D}_{0}$. Besides the charge transfer, the model system (1) - 4) accounts for electrostatic interaction between charges in $\mathcal{S}_{\mathrm{cl}}$ and $\mathcal{S}_{\mathrm{qm}}$.

In order to ensure local charge conservation $\nabla \cdot \mathbf{j}_{\text {tot }}=0$, the (net-)capture rates appearing in the carrier transport equations (2) and (3) are identified as

$$
\begin{aligned}
& S_{n}=+w(\mathbf{r}) \operatorname{tr}\left(N \mathcal{D}_{e}(\rho ; n, p, \psi)\right), \\
& S_{p}=-w(\mathbf{r}) \operatorname{tr}\left(N \mathcal{D}_{h}(\rho ; n, p, \psi)\right) .
\end{aligned}
$$

The (net-)capture rates $S_{n / p}$ contain all microscopic capture processes connected with transitions between the various multi-particle configurations of the $\mathrm{QD}$.

For different choices of $Q(\rho)$, e.g. different localization profiles of captured electrons and holes $Q(\rho)=$ $w_{h}(\mathbf{r}) \operatorname{tr}\left(n_{h} \rho\right)-w_{e}(\mathbf{r}) \operatorname{tr}\left(n_{e} \rho\right)$ (with $w_{e / h}$ normalized), the property of local charge conservation is lost in general. However, the violation of local charge conservation is restricted to a small region $\nabla \cdot \mathbf{j}_{\text {tot }} \propto\left(w_{e}(\mathbf{r})-w_{h}(\mathbf{r})\right)$ and is preserved globally, i.e. it holds $\int_{\Omega} \mathrm{d}^{3} r \nabla \cdot \mathbf{j}_{\text {tot }}=0$.

The thermodynamic consistency discussed in the subsequent sections does not crucially rely on the property of local charge conservation as enforced by Eq. (14). With some minor modifications, the approach can be generalized to cases where only the weaker condition of global charge conservation is fulfilled. This allows e.g. for capture rates with a more complicated spatial dependency than the one stated in Eq. (14). Since the discussion of thermodynamic consistency is least technical in the case of local charge conservation, we assume Eq. (14) in the following. Other cases can be treated analogously.

\section{THERMODYNAMICS}

In the recent years, the on-going miniaturization of (quantum) electronic devices has enabled the investiga- 
tion of thermodynamical laws on the nanoscale. This has lead to the emergence of the novel field of quantum thermodynamics [38 41]. Experiments and theory indicate that the fundamental thermodynamical laws also hold in the quantum regime [42, 43] and therefore we view thermodynamic consistency as a crucial feature for any hybrid quantum-classical model.

In this section we discuss the thermodynamic properties of the hybrid model system (1)-(4). At first, this concerns a consideration of the energy, charge and entropy balance between the system and its reservoirs. Second, the thermodynamic equilibrium solution of the hybrid system will be constructed by minimizing its grand potential. Moreover, we formulate a relation between the microscopic transition rates satisfying the quantum detailed balance condition. Finally, the hybrid quantumclassical model (1)-(4) is shown to have a non-negative entropy production rate, which we interpret as consistency with the second law of thermodynamics.

\section{A. Energy, charge and entropy balance}

We consider an open system $\mathcal{S}$, which itself consists of a classical subsystem $\mathcal{S}_{\mathrm{cl}}$ and quantum-mechanical subsystem $\mathcal{S}_{\mathrm{qm}}$. The system $\mathcal{S}$ is in contact with several reservoirs $\mathcal{R}_{i}$ as illustrated in Fig. 2(a). The system $\mathcal{S}$ can exchange energy and charge carriers with the reservoirs. The combined system is assumed to be isolated. The reservoir $\mathcal{R}_{0}$ is a heat bath with fixed background temperature $T$, which comprises the crystal lattice as well as the surrounding radiation field. The reservoirs $\mathcal{R}_{i \geq 1}$ model the electrical contacts at the boundary of the device. They are characterized by a common temperature and their chemical potentials $\mu_{i}$ (or applied voltages), which enter the system (1)-(4) via boundary conditions (cf. Appendix A).

The total change of entropy is given by

$$
\Delta S_{\text {tot }}=\Delta S_{\mathcal{S}}+\Delta S_{\mathcal{R}} \geq 0
$$

and the conservation of the total internal energy and charge is expressed as

$$
\begin{aligned}
& \Delta U=\Delta U_{\mathcal{S}}+\Delta U_{\mathcal{R}}=0, \\
& \Delta N=\Delta N_{\mathcal{S}}+\Delta N_{\mathcal{R}}=0 .
\end{aligned}
$$

The reservoir $\mathcal{R}_{0}$ can exchange only energy with $\mathcal{S}$, hence its change of entropy is given by $\Delta S_{\mathcal{R}_{0}}=\frac{1}{T} \Delta U_{\mathcal{R}_{0}}$. For the contacts $\mathcal{R}_{i \geq 1}$, also charge transfer is possible such that $\Delta S_{\mathcal{R}_{i \geq 1}}=\frac{1}{T} \Delta U_{\mathcal{R}_{i}}-\frac{\mu_{i}}{T} \Delta N_{\mathcal{R}_{i}}$. Using the conservation laws state above and $\Delta U_{\mathcal{R}}=\sum_{i \geq 0} \Delta U_{\mathcal{R}_{i}}$, we obtain

$$
\Delta S_{\text {tot }}=\Delta S_{\mathcal{S}}-\frac{1}{T} \Delta U_{\mathcal{S}}-\sum_{i \geq 1} \frac{\mu_{i}}{T} \Delta N_{\mathcal{R}_{i}}
$$

where $\Delta N_{\mathcal{R}_{i}}$ is just the (negative) charge flow across the boundary $\Gamma_{i}$. Using

$$
\lim _{\Delta t \rightarrow 0} \frac{\Delta N_{\mathcal{R}_{i}}}{\Delta t}=\frac{\mathrm{d} N_{\mathcal{R}_{i}}}{\mathrm{~d} t}=-\frac{1}{q} \int_{\Gamma_{i}} \mathrm{~d} \mathbf{A} \cdot\left(\mathbf{j}_{n}+\mathbf{j}_{p}\right),
$$

we obtain the entropy production rate

$$
\frac{\mathrm{d} S_{\text {tot }}}{\mathrm{d} t}=-\frac{1}{T} \frac{\mathrm{d} F_{\mathcal{S}}}{\mathrm{d} t}+\sum_{i \geq 1} \frac{\mu_{i}}{q T} \int_{\Gamma_{i}} \mathrm{~d} \mathbf{A} \cdot\left(\mathbf{j}_{n}+\mathbf{j}_{p}\right),
$$

where $F_{\mathcal{S}}=U_{\mathcal{S}}-T S_{\mathcal{S}}$ denotes the free energy of the system $\mathcal{S}$. In Sec. IIID it will be shown, that the entropy production rate is indeed always positive for the hybrid model (1)- (4). Under chemical equilibrium boundary conditions (all reservoirs $\mathcal{R}_{i \geq 1}$ have the chemical potential $\mu_{i}=\mu_{\mathrm{eq}}$ ), the above expression simplifies further. Exploiting the conservation of total charge, one obtains

$$
\left.\frac{\mathrm{d} S_{\mathrm{tot}}}{\mathrm{d} t}\right|_{\mathrm{eq}}=-\frac{1}{T} \frac{\mathrm{d} \Omega_{\mathcal{S}}}{\mathrm{d} t}
$$

with the grand potential $\Omega_{\mathcal{S}}=U_{\mathcal{S}}-T S_{\mathcal{S}}-\mu_{\mathrm{eq}} N_{\mathcal{S}}$. Thus, $\Omega_{\mathcal{S}}$ is a Lyapunov function for the irreversible relaxation of $\mathcal{S}$ into the thermodynamic equilibrium.

\section{B. Thermodynamic equilibrium}

According to Eq. (16), the thermodynamic equilibrium solution of (1)-(4) can be constructed by minimizing the grand potential $\Omega_{\mathcal{S}}$. Since we assume only a weak coupling between the quantum system and its macroscopic environment, the total entropy, total internal energy and total charge number are given by sums of the classical and the quantum mechanical contribution

$$
\begin{aligned}
S(n, p, \rho)= & S_{\mathrm{cl}}(n, p)+S_{\mathrm{qm}}(\rho), \\
U(n, p, \rho)= & U_{\mathrm{cl}}(n, p)+U_{\mathrm{qm}}(\rho) \\
& +U_{\psi}(p-n+Q(\rho)), \\
N(n, p, \rho)= & N_{\mathrm{cl}}(n, p)+N_{\mathrm{qm}}(\rho) .
\end{aligned}
$$

Here also the energy contribution $U_{\psi}$ of the electrostatic field is taken into account. The extensive thermodynamic quantities of the macroscopic system are expressed via volume densities

$$
\begin{aligned}
& S_{\mathrm{cl}}(n, p)=\int_{\Omega} \mathrm{d}^{3} r s_{\mathrm{cl}}(n, p), \\
& U_{\mathrm{cl}}(n, p)=\int_{\Omega} \mathrm{d}^{3} r u_{\mathrm{cl}}(n, p), \\
& N_{\mathrm{cl}}(n, p)=\int_{\Omega} \mathrm{d}^{3} r(n-p)
\end{aligned}
$$

with the entropy density $s_{\mathrm{cl}}$ and the internal energy density $u_{\mathrm{cl}}$. We consider the continuum carriers to be in a local thermodynamic equilibrium 33. Hence, the internal energy density and the entropy density can be expressed 
as functions of the local carrier density

$$
\begin{aligned}
s_{\mathrm{cl}}= & -k_{B}\left(n F_{1 / 2}^{-1}\left(\frac{n}{N_{c}}\right)-\frac{5}{2} N_{c} F_{3 / 2}\left(F_{1 / 2}^{-1}\left(\frac{n}{N_{c}}\right)\right)\right) \\
& -k_{B}\left(p F_{1 / 2}^{-1}\left(\frac{p}{N_{v}}\right)-\frac{5}{2} N_{v} F_{3 / 2}\left(F_{1 / 2}^{-1}\left(\frac{p}{N_{v}}\right)\right)\right), \\
u_{\mathrm{cl}}= & \frac{3}{2} k_{B} T N_{c} F_{3 / 2}\left(F_{1 / 2}^{-1}\left(\frac{n}{N_{c}}\right)\right)+E_{c} n \\
& +\frac{3}{2} k_{B} T N_{v} F_{3 / 2}\left(F_{1 / 2}^{-1}\left(\frac{p}{N_{v}}\right)\right)-E_{v} p .
\end{aligned}
$$

The above relations are obtained for the quasi-free electron and hole gas with parabolic energy dispersion and Fermi-Dirac statistics in three dimensions [44]. The contributions of the quantum system are given by the von Neumann entropy and the expectation values of the Hamiltonian $H$ and the charge number operator $N$

$$
\begin{aligned}
S_{\mathrm{qm}} & =-k_{B} \operatorname{tr}(\rho \log \rho), \\
U_{\mathrm{qm}} & =\operatorname{tr}(H \rho), \\
N_{\mathrm{qm}} & =\operatorname{tr}(N \rho) .
\end{aligned}
$$

The carriers interact via their self-consistently generated electrostatic field, which yields the contribution $U_{\psi}$ to the internal energy. It is convenient to decompose the total electrostatic potential into $\psi=\psi_{\text {int }}+\psi_{\text {ext }}$, where the internal field $\psi_{\text {int }}=\psi_{\text {int }}\left(\rho_{\text {int }}\right)$ is generated by the total internal carrier density

$$
\rho_{\text {int }}=p-n+Q(\rho),
$$

whereas the external field $\psi_{\text {ext }}$ arises from the built-in doping profile and voltages applied at the electric contacts. Then, the field energy can be written as 45

$$
U_{\psi}\left(\rho_{\text {int }}\right)=\frac{1}{2} \int_{\Omega} \mathrm{d}^{3} r \varepsilon\left|\nabla \psi_{\text {int }}\left(\rho_{\text {int }}\right)\right|^{2}+q \int_{\Omega} \mathrm{d}^{3} r \rho_{\text {int }} \psi_{\text {ext }} .
$$

Assuming the charge density of the quantum system as stated in Eq. 13), and finally minimizing the grand potential $\Omega_{\mathcal{S}}$ under the constraint $\operatorname{tr}(\rho)=1$, we obtain the equilibrium free carrier densities as

$$
\begin{aligned}
& n_{\mathrm{eq}}=N_{c} F_{1 / 2}\left(\beta\left(\mu_{\mathrm{eq}}-E_{c}+q \psi_{\mathrm{eq}}\right)\right), \\
& p_{\mathrm{eq}}=N_{v} F_{1 / 2}\left(\beta\left(E_{v}-q \psi_{\mathrm{eq}}-\mu_{\mathrm{eq}}\right)\right)
\end{aligned}
$$

and the equilibrium density matrix

$$
\rho_{\mathrm{eq}}=\frac{1}{Z} e^{-\beta\left(H-\left(\mu_{\mathrm{eq}}+q\left\langle\psi_{\mathrm{eq}}\right\rangle_{w}\right) N\right)} .
$$

Here, $Z=\operatorname{tr}\left(\exp \left(-\beta\left(H-\left(\mu_{\mathrm{eq}}+q\left\langle\psi_{\mathrm{eq}}\right\rangle_{w}\right) N\right)\right)\right)$ represents the grand canonical partition function,

$$
\langle\psi\rangle_{w}=\int_{\Omega} \mathrm{d}^{3} r w(\mathbf{r}) \psi(\mathbf{r})
$$

is the averaged electrostatic potential in the vicinity of the QD and the built-in potential $\psi_{\text {eq }}$ solves Eq. (1) with the right hand side $q\left(p_{\text {eq }}-n_{\text {eq }}+C+Q\left(\rho_{\text {eq }}\right)\right)$ at equilibrium boundary conditions. The equilibrium density matrix is a grand canonical ensemble, which contains a contribution from the electrostatic potential due to the electrostatic interaction with the macroscopic environment. The latter appears in Eq. (21) as a spatial average using the localization profile $w$ of the confined carriers as a weighting function, see Eq. (22). This is a remarkable result, which indicates that the quantum system interacts only with its spatially averaged macroscopic environment. We emphasize that this is a direct consequence of the ansatz Eq. (13) and the variation of Eq. (20) with respect to $n, p$ and $\rho$. See Appendix $\mathrm{B}$ for details.

In the following, the concept of a non-local interaction of the quantum system with its spatially averaged macroscopic environment will be extended to non-equilibrium situations.

\section{Microscopic transition rates and the quantum detailed balance condition}

We assume the microscopic transition rates in the dissipator $(9)$ to be functions of the spatially averaged macroscopic potentials

$$
\begin{aligned}
& \gamma_{\alpha}=\gamma_{\alpha}\left(\left\langle\mu_{c}\right\rangle_{w},\left\langle\mu_{v}\right\rangle_{w},\langle\psi\rangle_{w}\right), \\
& \hat{\gamma}_{\alpha}=\hat{\gamma}_{\alpha}\left(\left\langle\mu_{c}\right\rangle_{w},\left\langle\mu_{v}\right\rangle_{w},\langle\psi\rangle_{w}\right),
\end{aligned}
$$

where $\langle\cdot\rangle_{w}$ denotes the spatial average according to Eq. (22). The quantum detailed balance condition requires the dissipator to vanish in equilibrium. Hence, the condition

$0 \stackrel{!}{=} \mathcal{D}_{\alpha}\left(\rho_{\text {eq }} ; n_{\text {eq }}, p_{\text {eq }}, \psi_{\text {eq }}\right)=\gamma_{\alpha}^{\mathrm{eq}} L_{A_{\alpha}}\left(\rho_{\text {eq }}\right)+\hat{\gamma}_{\alpha}^{\mathrm{eq}} L_{A_{\alpha}^{\dagger}}\left(\rho_{\text {eq }}\right)$

can be used to derive a relation between the equilibrium transition rates $\gamma_{\alpha}^{\mathrm{eq}}=\gamma_{\alpha}\left(\mu_{\mathrm{eq}}, \mu_{\mathrm{eq}},\left\langle\psi_{\mathrm{eq}}\right\rangle_{w}\right)$ and $\hat{\gamma}_{\alpha}^{\mathrm{eq}}$. From Eq. 10, one obtains for any $\lambda \in \mathbb{R}$

$$
\begin{aligned}
& e^{\lambda H} A_{\alpha} e^{-\lambda H}=e^{-\lambda \hbar \omega_{\alpha}} A_{\alpha}, \\
& e^{\lambda N} A_{\alpha} e^{-\lambda N}=e^{-\lambda \ell_{\alpha}} A_{\alpha},
\end{aligned}
$$

which implies

$$
\begin{aligned}
& A_{\alpha} \rho_{\mathrm{eq}}=e^{-\beta\left(\hbar \omega_{\alpha}-\left(\mu_{\mathrm{eq}}+q\left\langle\psi_{\mathrm{eq}}\right\rangle_{w}\right) \ell_{\alpha}\right)} \rho_{\mathrm{eq}} A_{\alpha}, \\
& A_{\alpha}^{\dagger} \rho_{\mathrm{eq}}=e^{+\beta\left(\hbar \omega_{\alpha}-\left(\mu_{\mathrm{eq}}+q\left\langle\psi_{\mathrm{eq}}\right\rangle_{w}\right) \ell_{\alpha}\right)} \rho_{\mathrm{eq}} A_{\alpha}^{\dagger} .
\end{aligned}
$$

Subsequently, one obtains

$$
\begin{aligned}
0 \stackrel{!}{=} & \left(\gamma_{\alpha}^{\mathrm{eq}}-\hat{\gamma}_{\alpha}^{\mathrm{eq}} e^{+\beta\left(\hbar \omega_{\alpha}-\left(\mu_{\mathrm{eq}}+q\left\langle\psi_{\mathrm{eq}}\right\rangle_{w}\right) \ell_{\alpha}\right)}\right) \times \\
& \times\left(A_{\alpha} \rho_{\mathrm{eq}} A_{\alpha}^{\dagger}-e^{-\beta\left(\hbar \omega_{\alpha}-\left(\mu_{\mathrm{eq}}+q\left\langle\psi_{\mathrm{eq}}\right\rangle_{w}\right) \ell_{\alpha}\right)} A_{\alpha}^{\dagger} \rho_{\mathrm{eq}} A_{\alpha}\right),
\end{aligned}
$$

which yields the desired relation between $\gamma_{\alpha}^{\text {eq }}$ and $\hat{\gamma}_{\alpha}^{\text {eq }}$ :

$$
\hat{\gamma}_{\alpha}^{\mathrm{eq}}=\gamma_{\alpha}^{\mathrm{eq}} e^{-\beta\left(\hbar \omega_{\alpha}-\left(\mu_{\mathrm{eq}}+q\left\langle\psi_{\mathrm{eq}}\right\rangle_{w}\right) \ell_{\alpha}\right)} .
$$


This agrees with the relation imposed by the KuboMartin-Schwinger (KMS) condition on the equilibrium reservoir correlation functions [28, 36]. Since throughout this paper we consider only thermalized environments, we extend the above relation to non-equilibrium situations

$$
\begin{aligned}
& \hat{\gamma}_{\alpha}\left(\left\langle\mu_{c}\right\rangle_{w},\left\langle\mu_{v}\right\rangle_{w},\langle\psi\rangle_{w}\right)= \\
& \quad=e^{-\beta\left(\hbar \omega_{\alpha}-\left(\left\langle\mu_{\alpha}\right\rangle_{w}+q\langle\psi\rangle_{w}\right) \ell_{\alpha}\right)} \gamma_{\alpha}\left(\left\langle\mu_{c}\right\rangle_{w},\left\langle\mu_{v}\right\rangle_{w},\langle\psi\rangle_{w}\right)
\end{aligned}
$$

with $\mu_{\alpha \in I_{e}}=\mu_{c}$ and $\mu_{\alpha \in I_{h}}=\mu_{v}$. For charge-conserving processes we require $\ell_{\alpha \in I_{0}}=0$, single electron-capture processes are described by $\ell_{\alpha \in I_{e}}=-1$ and for single hole-capture processes it holds $\ell_{\alpha \in I_{h}}=+1$.

Thus, supposing Eq. 23), the hybrid model obeys the quantum detailed balance condition for any model of the forward transition rate $\gamma_{\alpha}\left(\left\langle\mu_{c}\right\rangle_{w},\left\langle\mu_{v}\right\rangle_{w},\langle\psi\rangle_{w}\right) \geq 0$ that is non-negative. Physically, the latter one must represent a parametrization of a microscopically derived transition rate (using Fermi's Golden Rule [46]) in terms of the averaged macroscopic potentials. In particular, this enables the direct inclusion of microscopically calculated capture rates e.g. from Refs. 47 51].

\section{Entropy production and the second law of thermodynamics}

From Eq. 15 we obtain the entropy production rate as (see Appendix $\mathrm{C}$ for the derivation)

$$
\begin{aligned}
\frac{\mathrm{d} S_{\text {tot }}}{\mathrm{d} t}= & \frac{1}{T} \int_{\Omega} \mathrm{d}^{3} r\left(\mu_{c}-\mu_{v}\right) R \\
& +\frac{1}{q T} \int_{\Omega} \mathrm{d}^{3} r\left(\mathbf{j}_{n} \cdot \nabla \mu_{c}+\mathbf{j}_{p} \cdot \nabla \mu_{v}\right) \\
& -k_{B} \operatorname{tr}\left((\beta H+\log \rho) \mathcal{D}_{0}\left(\rho ; \chi_{w}\right)\right) \\
& -k_{B} \operatorname{tr}\left(\left(\beta\left(H-\mu_{c}^{\text {eff }} N\right)+\log \rho\right) \mathcal{D}_{e}\left(\rho ; \chi_{w}\right)\right) \\
& -k_{B} \operatorname{tr}\left(\left(\beta\left(H-\mu_{v}^{\text {eff }} N\right)+\log \rho\right) \mathcal{D}_{h}\left(\rho ; \chi_{w}\right)\right)
\end{aligned}
$$

with $\mu_{c / v}^{\mathrm{eff}}=\left\langle\mu_{c / v}\right\rangle_{w}+q\langle\psi\rangle_{w}$. The dependency of the dissipators on the state of the classical environment is indicated by the abbreviation $\chi_{w}=\left(\left\langle\mu_{c}\right\rangle_{w},\left\langle\mu_{v}\right\rangle_{w},\langle\psi\rangle_{w}\right)$. The first two lines describe the entropy production rate of the van Roosbroeck system [52] and the third line is the entropy production rate of an open quantum system coupled to a heat bath [53]. The fourth and fifth line represent the contributions arising from the coupling of the QD with its macroscopic environment via capture and escape. All terms are products of abstract thermodynamic forces and their corresponding fluxes, which is in agreement with the general theory of linear irreversible thermodynamics [33. Using Spohn's inequality [53, it can be shown that all individual lines of Eq. 24) are non-negative and therefore it holds

$$
\frac{\mathrm{d} S_{\text {tot }}}{\mathrm{d} t} \geq 0,
$$

where the equality holds only in the case of thermodynamic equilibrium. A proof is given in the Appendix $\mathrm{D}$.

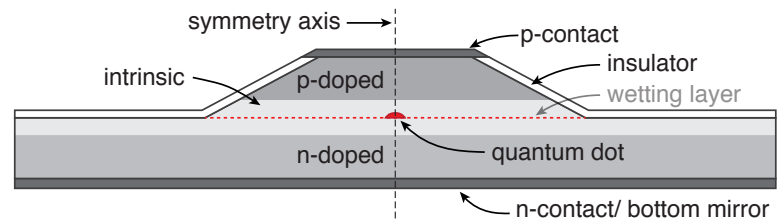

Figure 3. Cross section of the example device considered in the numerical simulations: A single QD is placed on the symmetry axis within the center of the intrinsic zone of a cylindrical $\mathrm{p}-\mathrm{i}-\mathrm{n}$ diode with etched mesa structure on top. The bottom mirror leads to a directed emission in vertical direction. Due to the lack of a top mirror, the device represents a leaky photonic cavity with low $Q$ factor. The device has electric contacts at the top and the bottom facets.

This results relies on the specific coupling imposed in the previous sections, which involves the spatially averaged macroscopic potentials. We emphasize, that if e.g. averaged carrier densities were used instead, a nonnegative entropy production rate could not be guaranteed in general. Finally, we conclude that our hybrid quantum-classical modeling approach is consistent with the second law of thermodynamics.

Our approach can also be interpreted as a damped Hamiltonian system in the framework of GENERIC (general equation for the non-equilibrium reversibleirreversible coupling) [54], which automatically ensures a non-negative entropy production rate and the existence of an unique thermodynamic equilibrium. It can be applied to a wide range of physical problems [55 57.

\section{APPLICATION TO ELECTRICALLY DRIVEN SINGLE-PHOTON SOURCES}

In this section we demonstrate the usefulness of our approach for applications in semiconductor device simulation. As an example we consider an electrically driven single-photon source based on a p-i-n diode including a single QD. Such devices have been shown to act as singlephoton emitters and are promising candidates for applications in quantum communication networks [58 61].

\section{A. Model specification}

The model equations are described in Sec. II] and III. For the hybrid system (1)-(4), we have to specify the Hamiltonian $H$ as well as the quantum jump operators $A_{\alpha}$ and the transition rates $\gamma_{\alpha}$, which constitute the dissipative interactions with the macroscopic environment. In particular, they need to satisfy the conditions (8) and (12) that guarantee charge conservation and the eigenoperator relations 100 . 


\section{Hamiltonian}

We consider a single QD embedded in a very leaky dielectric cavity with low $Q$ factor, which is sketched in Fig. 3. In such devices, the light-matter interaction is governed by spontaneous emission and thus can be described by a Lindblad dissipator. Hence, we can model the quantum system by a purely electronic Hamiltonian. We aim for a description of the electronic QD states in terms of many-body states covering single particle states, excitons, trions and the biexciton as shown in Fig. 4(a). We assume a single one-particle level (ground state) for the electrons and holes each, labeled by $\varepsilon_{c}$ and $\varepsilon_{v}$, respectively. The Hamiltonian

$$
H=H_{0}+H_{I}
$$

contains the single-particle contributions

$$
H_{0}=\sum_{\sigma} \varepsilon_{c} e_{\sigma}^{\dagger} e_{\sigma}-\sum_{\sigma} \varepsilon_{v} h_{\sigma}^{\dagger} h_{\sigma}
$$

and their Coulomb interaction

$$
\begin{gathered}
H_{I}=\frac{1}{2} \sum_{\sigma, \sigma^{\prime}}\left(V_{c, c} e_{\sigma}^{\dagger} e_{\sigma^{\prime}}^{\dagger} e_{\sigma^{\prime}} e_{\sigma}+V_{v, v} h_{\sigma}^{\dagger} h_{\sigma^{\prime}}^{\dagger} h_{\sigma^{\prime}} h_{\sigma}-\right. \\
\left.-2 V_{c, v} e_{\sigma}^{\dagger} h_{\sigma^{\prime}}^{\dagger} h_{\sigma^{\prime}} e_{\sigma}\right) .
\end{gathered}
$$

The operators $e_{\sigma}^{\dagger}\left(e_{\sigma}\right)$ and $h_{\sigma}^{\dagger}\left(h_{\sigma}\right)$ create (annihilate) an electron or hole with total angular momentum quantum number in $z$-direction $\sigma$. We consider a single valence band describing heavy holes with a pseudo spin $\pm 3 / 2$ indicated by $\{\Uparrow, \Downarrow\}$. Here, only Hartree-like Coulomb matrix elements $V_{i, j}=V_{i, j, j, i}$ occur, which are of the order of several tens of meV (see Appendix F). The creation and annihilation operators obey the fermionic anticommutator relations $\left\{e_{\sigma}, e_{\sigma^{\prime}}^{\dagger}\right\}=\left\{h_{\sigma}, h_{\sigma^{\prime}}^{\dagger}\right\}=\delta_{\sigma, \sigma^{\prime}}$ and $\left\{e_{\sigma}, e_{\sigma^{\prime}}\right\}=\left\{h_{\sigma}, h_{\sigma^{\prime}}\right\}=0$. The single-particle energy levels and the Coulomb matrix elements are obtained from Schrödinger's equation with an effective confinement potential for InGaAs-QDs 62. With the number operators $n_{e, \sigma}=e_{\sigma}^{\dagger} e_{\sigma}, n_{h, \sigma}=h_{\sigma}^{\dagger} h_{\sigma}$ and the abbreviations

$$
n_{e}=\sum_{\sigma=\{\uparrow, \downarrow\}} n_{e, \sigma}, \quad n_{h}=\sum_{\sigma=\{\Uparrow, \Downarrow\}} n_{h, \sigma},
$$

we can express the Hamiltonian in the occupation number representation as

$$
\begin{aligned}
H= & \left(\varepsilon_{c}-\frac{1}{2} V_{c, c}\right) n_{e}-\left(\varepsilon_{v}+\frac{1}{2} V_{v, v}\right) n_{h} \\
& +\frac{1}{2} V_{c, c} n_{e}^{2}+\frac{1}{2} V_{v, v} n_{h}^{2}-V_{c, v} n_{e} n_{h} .
\end{aligned}
$$

By diagonalization, we obtain the spectral representation of $H$ in terms of multi-particle states

$$
H=\sum_{k} \varepsilon_{k}|k\rangle\langle k|,
$$

where $k=\left(n_{e, \uparrow}, n_{e, \downarrow}, n_{h, \Uparrow}, n_{h, \Downarrow}\right)$ is a multi-index labeling the 16 different electronic configurations which are illustrated in Fig. 4(a, b). If excited states are included and full configuration interaction is taken into account, the diagonalization of $H$ is in general a non-trivial task. In this case, an approximative representation of the Coulomb interaction in terms of number operators as in Eq. 25 can be obtained by the Hartree-Fock approximation 63.

\section{Dissipators}

We describe the spontaneous emission and the capture and escape of carriers by dissipators of the type (9). Even though the Hamiltonian of the quantum system Eq. 25 has a degenerate energy spectrum (due to spin degeneracy), the coherences are decoupled from the populations because of the selection rules. Hence, the resulting dynamical system reduces to a master equation for the populations [37]. In this case, a jump operator $A_{\alpha}$ describes a transition between two multi-particle states $|i\rangle$ and $|f\rangle$ is given by the projector $|f\rangle\langle i|$. The allowed transitions are indicated by arrows in Fig. 4(a), e.g. the dissipator connected with $A_{\alpha}=\left|X_{1}\right\rangle\left\langle e^{\uparrow}\right|$ describes the capture of a hole into a QD occupied by a single electron leading to the formation of the bright exciton $\left|X_{1}\right\rangle$. By using adjacency matrices to encode the allowed transitions shown in Fig. 4(a), the dissipation superoperators for all processes can be written in a compact form as

$$
\begin{aligned}
\mathcal{D}_{e}\left(\rho ; \chi_{w}\right)= & \sum_{i, f} \mathcal{A}_{i, f}^{e} \gamma_{i \rightarrow f}^{e}\left(\chi_{w}\right) \times \\
& \times\left(L_{|f\rangle\langle i|}(\rho)+e^{-\beta \Delta \varepsilon_{i, f}^{e}\left(\chi_{w}\right)} L_{|i\rangle\langle f|}(\rho)\right), \\
\mathcal{D}_{h}\left(\rho ; \chi_{w}\right)= & \sum_{i, f} \mathcal{A}_{i, f}^{h} \gamma_{i \rightarrow f}^{h}\left(\chi_{w}\right) \times \\
& \times\left(L_{|f\rangle\langle i|}(\rho)+e^{-\beta \Delta \varepsilon_{i, f}^{h}\left(\chi_{w}\right)} L_{|i\rangle\langle f|}(\rho)\right), \\
\mathcal{D}_{0}(\rho)=\sum_{i, f} & \mathcal{A}_{i, f}^{0} \gamma_{i \rightarrow f}^{0} \times \\
& \times\left(L_{|f\rangle\langle i|}(\rho)+e^{\left.-\beta \Delta \varepsilon_{i, f}^{0} L_{|i\rangle\langle f|}(\rho)\right),}\right.
\end{aligned}
$$

where the indices $i$ and $f$ run over all multi-particle eigenstates. Again, the dependency of the dissipation superoperators on the state of the classical environment is indicated by the abbreviation $\chi_{w}=\left(\left\langle\mu_{c}\right\rangle_{w},\left\langle\mu_{v}\right\rangle_{w},\langle\psi\rangle_{w}\right)$. In accordance with Eq. 23, the effective transition energies are given as

$$
\begin{aligned}
\Delta \varepsilon_{i, f}^{e}\left(\chi_{w}\right) & =\varepsilon_{i}-\varepsilon_{f}-q\langle\psi\rangle_{w}-\left\langle\mu_{c}\right\rangle_{w}, \\
\Delta \varepsilon_{i, f}^{h}\left(\chi_{w}\right) & =\varepsilon_{i}-\varepsilon_{f}+q\langle\psi\rangle_{w}+\left\langle\mu_{v}\right\rangle_{w}, \\
\Delta \varepsilon_{i, f}^{0} & =\varepsilon_{i}-\varepsilon_{f}
\end{aligned}
$$

and the adjacency matrix elements encoding Pauli blocking and the optical selection rules (conservation of total 
angular momentum) read

$$
\begin{aligned}
\mathcal{A}_{i, f}^{e}= & \delta_{\left\langle i\left|n_{e}\right| i\right\rangle+1,\left\langle f\left|n_{e}\right| f\right\rangle} \prod_{\sigma=\{\Uparrow, \Downarrow\}} \delta_{\left\langle i\left|n_{h, \sigma}\right| i\right\rangle,\left\langle f\left|n_{h, \sigma}\right| f\right\rangle}, \\
\mathcal{A}_{i, f}^{h}= & \delta_{\left\langle i\left|n_{h}\right| i\right\rangle+1,\left\langle f\left|n_{h}\right| f\right\rangle} \prod_{\sigma=\{\uparrow, \downarrow\}} \delta_{\left\langle i\left|n_{e, \sigma}\right| i\right\rangle,\left\langle f\left|n_{e, \sigma}\right| f\right\rangle}, \\
\mathcal{A}_{i, f}^{0}= & \delta_{\left\langle i\left|n_{e, \uparrow}\right| i\right\rangle,\left\langle f\left|n_{e, \uparrow}\right| f\right\rangle} \delta_{\left\langle i\left|n_{e, \downarrow}\right| i\right\rangle-1,\left\langle f\left|n_{e, \downarrow}\right| f\right\rangle} \times \\
& \times \delta_{\left\langle i\left|n_{h, \Uparrow}\right| i\right\rangle-1,\left\langle f\left|n_{h, \Uparrow}\right| f\right\rangle} \delta_{\left\langle i\left|n_{h, \Downarrow}\right| i\right\rangle,\left\langle f\left|n_{h, \Downarrow}\right| f\right\rangle}+ \\
+ & \delta_{\left\langle i\left|n_{e, \uparrow}\right| i\right\rangle-1,\left\langle f\left|n_{e, \uparrow}\right| f\right\rangle} \delta_{\left\langle i\left|n_{e, \downarrow}\right| i\right\rangle,\left\langle f\left|n_{e, \downarrow}\right| f\right\rangle} \times \\
& \times \delta_{\left\langle i\left|n_{h, \Uparrow}\right| i\right\rangle,\left\langle f\left|n_{h, \Uparrow}\right| f\right\rangle} \delta_{\left\langle i\left|n_{h, \Downarrow}\right| i\right\rangle-1,\left\langle f\left|n_{h, \Downarrow}\right| f\right\rangle}
\end{aligned}
$$

Please note that the above adjacency matrices are nonsymmetric $\mathcal{A}_{i, f} \neq \mathcal{A}_{f, i}$. Thereby, they contain a directionality which refers to the primary processes indicated by the arrow directions shown in Fig. 4(a). This is employed in the notation of the dissipators in Eq. (26), which explicitly accounts for the actual net-transition rates by hard-wiring the relation (23) between forward and backward rates. Consequently, the quantum detailed balance relation is guaranteed in the thermodynamic equilibrium independent of the model for the forward rate $\gamma_{i \rightarrow f}\left(\chi_{w}\right)$. The respective backward transition rate is obtained according to Eq. 23. An alternative representation of the master equation for the populations can be found in Appendix E

\section{Transition rate models}

The spontaneous decay rates of the various (bright) electronic states of the quantum system can be modeled by the Weisskopf-Wigner rate [64]

$$
\gamma_{i \rightarrow f}^{0}=\frac{P_{i, f} d_{c, v}^{2} n_{r}}{6 \pi \hbar \varepsilon_{0} c_{0}^{3}}\left(\frac{\varepsilon_{i}-\varepsilon_{f}}{\hbar}\right)^{3}\left(1+n_{\mathrm{pt}}\left(\frac{\varepsilon_{i}-\varepsilon_{f}}{\hbar}\right)\right)
$$

for allowed index pairs $i \rightarrow f$ giving $\mathcal{A}_{i, f}^{0}=1$. Here, $n_{\mathrm{pt}}(\omega)=\left(e^{\beta \hbar \omega}-1\right)^{-1}$ is the thermally induced photon number, $n_{r}$ is the refractive index of the material, $d_{c, v}$ denotes the interband dipole moment and $c_{0}$ is the vacuum speed of light. Due to cavity effects, the decay rate is slightly modified with respect to the free space decay rate, which is accounted for by the Purcell factors $P_{i, f}$. The Weisskopf-Wigner rate is applicable in low $Q$ optical resonators, where the photonic density of states varies insignificantly over the linewidth of the emitter [15, 65. Using the parameters given in Appendix $\mathrm{F}$, all decay rates are found to be approximately $10^{9} \mathrm{~s}^{-1}$.

For semiconductor QDs, the Fröhlich coupling and Auger scattering typically constitute the dominant capture processes. As a rule of thumb, at low carrier densities, the LO-phonon assisted Fröhlich coupling provides the dominant scattering channel, whereas at elevated carrier densities the Auger scattering becomes increasingly efficient [9, 50, 66]. Due to the relatively large Coulomb
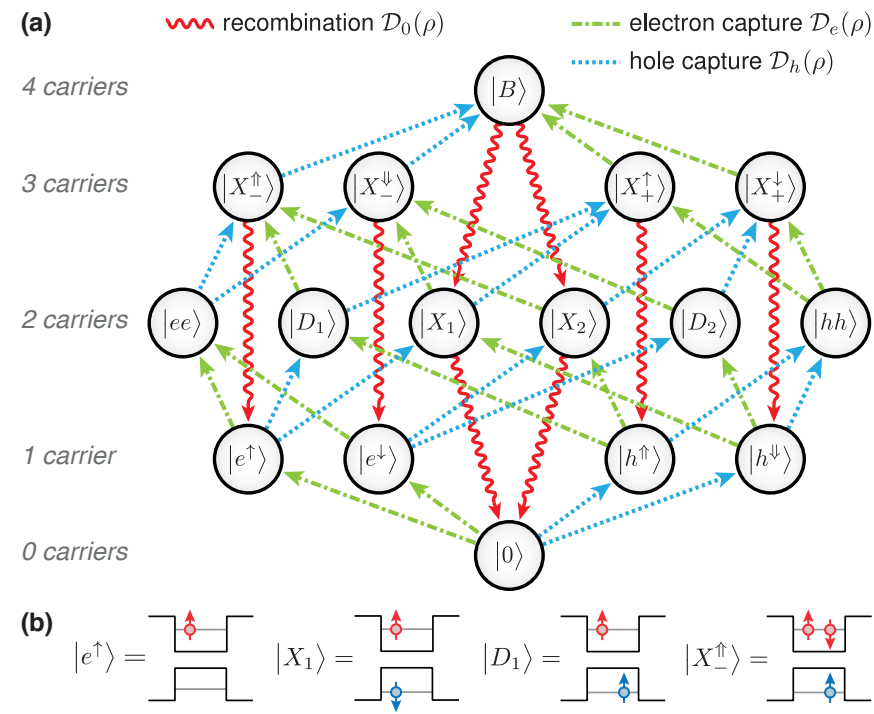

(c)

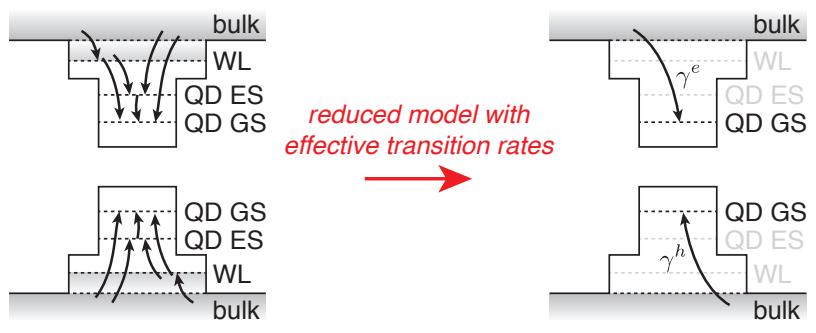

Figure 4. (a) Diagram of electronic states of the QDHamiltonian 25 and possible (irreversible) transitions. The arrows indicate capture and recombination, for the corresponding reverse processes (escape, generation) the arrows need to be reversed. We use short notations for the multi-particle states $\left|n_{e, \uparrow}, n_{e, \downarrow}, n_{h, \Uparrow}, n_{h, \Downarrow}\right\rangle$ : empty QD $|0\rangle=|0,0,0,0\rangle$, single-electron states $\left|e^{\uparrow}\right\rangle=|1,0,0,0\rangle$, $\left|e^{\downarrow}\right\rangle=|0,1,0,0\rangle$, single-hole states $\left|h^{\Uparrow}\right\rangle=|0,0,1,0\rangle,\left|h^{\Downarrow}\right\rangle=$ $|0,0,0,1\rangle$, two-electron state $|e e\rangle=|1,1,0,0\rangle$, two-hole state $|h h\rangle=|0,0,1,1\rangle$, bright excitons $\left|X_{1}\right\rangle=|1,0,0,1\rangle,\left|X_{2}\right\rangle=$ $|0,1,1,0\rangle$, dark excitons $\left|D_{1}\right\rangle=|1,0,1,0\rangle,\left|D_{2}\right\rangle=|0,1,0,1\rangle$, negative trions $\left|X_{-}^{\Uparrow}\right\rangle=|1,1,1,0\rangle,\left|X_{-}^{\Downarrow}\right\rangle=|1,1,0,1\rangle$, positive trions $\left|X_{+}^{\uparrow}\right\rangle=|1,0,1,1\rangle,\left|X_{+}^{\downarrow}\right\rangle=|0,1,1,1\rangle$ and the biexciton state $|B\rangle=|1,1,1,1\rangle$. (b) Schematic representation of the QD occupation for some example states. (c) Illustration of the effective scattering cascade in the reduced model involving only the single-particle ground states.

matrix elements in semiconductor QDs, the scattering rates into charged states differ significantly from those into neutral states. This effect is known as Coulomb suppression or Coulomb enhancement, respectively [66].

The scattering rates can be calculated microscopically by Fermi's Golden rule and then always satisfy the detailed balance relation between the forward and backward process [47 51]. However, here we restrict ourselves to phenomenological laws for the effective capture rate of continuum carriers into the QD. The respective escape rates follow via the detailed balance relation. The effective capture rate approximates the entire scattering 
cascade, see Fig. 4(c). We model the effective electron capture rates entering Eq. (26a) as

$$
\begin{aligned}
\gamma_{i \rightarrow f}^{e} & \left(\left\langle\mu_{c}\right\rangle_{w},\left\langle\mu_{v}\right\rangle_{w},\langle\psi\rangle_{w}\right)= \\
= & \frac{1+n_{\mathrm{LO}}}{\tau_{\mathrm{LO}}^{e}} \frac{1}{e^{\beta\left(E_{c}-q\langle\psi\rangle_{w}-\left\langle\mu_{c}\right\rangle_{w}+a_{\mathrm{LO}}^{e}+C_{i, f}^{e}\right)}+1}+ \\
& +\frac{1}{\tau_{\mathrm{Au}}^{e, e}} \frac{\bar{n}_{w}^{2}}{1+\bar{n}_{w}^{2-\gamma_{\mathrm{Au}}^{e, e}}}+\frac{1}{\tau_{\mathrm{Au}}^{e, h}} \frac{\bar{n}_{w} \bar{p}_{w}}{1+\left(\bar{n}_{w} \bar{p}_{w}\right)^{1-\gamma_{\mathrm{Au}}^{e, h} / 2}},
\end{aligned}
$$

for the admitted index pairs $i \rightarrow f$ giving $\mathcal{A}_{i, f}^{e}=1$ and the effective hole capture rates in Eq. (26b) as

$$
\begin{aligned}
\gamma_{i \rightarrow f}^{h} & \left(\left\langle\mu_{c}\right\rangle_{w},\left\langle\mu_{v}\right\rangle_{w},\langle\psi\rangle_{w}\right)= \\
= & \frac{1+n_{\mathrm{LO}}}{\tau_{\mathrm{LO}}^{h}} \frac{1}{e^{-\beta\left(E_{v}-q\langle\psi\rangle_{w}-\left\langle\mu_{v}\right\rangle-a_{\mathrm{LO}}^{h}-C_{i, f}^{h}\right)}+1}+ \\
& +\frac{1}{\tau_{\mathrm{Au}}^{h, h}} \frac{\bar{p}_{w}^{2}}{1+\bar{p}_{w}^{2-\gamma_{\mathrm{Au}}^{h, h}}}+\frac{1}{\tau_{\mathrm{Au}}^{h, e}} \frac{\bar{n}_{w} \bar{p}_{w}}{1+\left(\bar{n}_{w} \bar{p}_{w}\right)^{1-\gamma_{\mathrm{Au}}^{h,} / 2}}
\end{aligned}
$$

for the admitted index pairs $i \rightarrow f$ yielding $\mathcal{A}_{i, f}^{h}=1$. Here we have used the abbreviations $\bar{n}_{w}=n_{w} / n_{\mathrm{Au}}^{\text {crit }}$ and $\bar{p}_{w}=p_{w} / p_{\mathrm{Au}}^{\text {crit }}$. Please note that the ambient continuum carrier densities $n_{w}=N_{c} F_{1 / 2}\left(\beta\left(\left\langle\mu_{c}\right\rangle_{w}-E_{c}+q\langle\psi\rangle_{w}\right)\right)$ and $p_{w}=N_{v} F_{1 / 2}\left(\beta\left(E_{v}-q\langle\psi\rangle_{w}-\left\langle\mu_{v}\right\rangle_{w}\right)\right)$ are functions of the averaged macroscopic potentials. The first terms in Eq. 28 describe the LO-phonon assisted relaxation of continuum carriers and the last lines are each attributed to Auger scattering. The number of thermally excited LO-phonons is given by $n_{\mathrm{LO}}=\left(e^{\beta \hbar \omega_{\mathrm{LO}}}-1\right)^{-1}$. The time constants $\tau_{\mathrm{LO}}^{\lambda}$ and the parameters $a_{\mathrm{LO}}^{\lambda}, \gamma_{\mathrm{LO}}^{\lambda}$, $\lambda \in\{e, h\}$ are considered as fitting factors that can be extracted from microscopic calculations or experimental data. The phonon assisted capture rates involve the Coulomb enhancement/suppression factors

$$
\begin{aligned}
& C_{i, f}^{e}=\varepsilon_{f}-\varepsilon_{i}-\varepsilon_{c}, \\
& C_{i, f}^{h}=\varepsilon_{f}-\varepsilon_{i}+\varepsilon_{v},
\end{aligned}
$$

which describe the additional attractive or repulsive Coulomb shifts and thereby either enhance (if $C_{i, f}^{\lambda}<0$, $\lambda \in\{e, h\}$ ) or decrease (if $C_{i, f}^{\lambda}>0, \lambda \in\{e, h\}$ ) the capture rate. At low temperatures the effect of Coulomb enhancement or suppression becomes increasingly important. For the Auger-like capture processes the modifications of the capture rates due to Coulomb shifts are assumed to be negligible due to strong screening effects at high carrier densities. The expressions in Eq. (28) take saturation effects at high carrier densities into account. The functional form is motivated from microscopically computed results presented in Refs. [51, 66]. In the low density limit (Maxwell-Boltzmann approximation) the capture rate models asymptotically take the form

$$
\begin{aligned}
\gamma_{i \rightarrow f}^{e} \approx & \frac{\left(n_{\mathrm{LO}}+1\right) e^{-\beta a_{\mathrm{LO}}^{e}}}{\tau_{\mathrm{LO}}^{e} N_{c}} e^{-\beta C_{i, f}^{e}} n_{w}^{\mathrm{MB}} \\
& +\frac{\left(\bar{n}_{w}^{\mathrm{MB}}\right)^{2}}{\tau_{\mathrm{Au}}^{e, e}}+\frac{\bar{n}_{w}^{\mathrm{MB}} \bar{p}_{w}^{\mathrm{MB}}}{\tau_{\mathrm{Au}}^{e, h}}, \\
\gamma_{i \rightarrow f}^{h} \approx & \frac{\left(n_{\mathrm{LO}}+1\right) e^{-\beta a_{\mathrm{LO}}^{h}}}{\tau_{\mathrm{LO}}^{h} N_{v}} e^{-\beta C_{i, f}^{h}} p_{w}^{\mathrm{MB}} \\
& +\frac{\left(\bar{p}_{w}^{\mathrm{MB}}\right)^{2}}{\tau_{\mathrm{Au}}^{h, h}}+\frac{\bar{n}_{w}^{\mathrm{MB}} \bar{p}_{w}^{\mathrm{MB}}}{\tau_{\mathrm{Au}}^{h, e}},
\end{aligned}
$$

showing a linear dependency on the continuum carrier density in the case of LO-phonon assisted capture and a quadratic dependency for the Auger capture processes. Moreover, the Coulomb enhancement and suppression effect becomes apparent in this form. The expression for $n_{w}^{\mathrm{MB}}$ is obtained by replacing $F_{1 / 2}(\cdot) \rightarrow \exp (\cdot)$ in the above definition of $n_{w}$ (analogous for $p_{w}^{\mathrm{MB}}$ ). The parameters $n_{\mathrm{Au}}^{\text {crit }}, p_{\mathrm{Au}}^{\text {crit }}$ and $\gamma_{\mathrm{Au}}^{\lambda, \lambda^{\prime}} \lambda, \lambda^{\prime} \in\{e, h\}$ are fitting factors.

\section{B. Numerical simulation method}

The van Roosbroeck system (1)-(3) is discretized using a Voronoï box based finite volumes method [32, 67] along with a modified Scharfetter-Gummel scheme 68 60 , for the discretization of the current densities. The latter one properly reflects the strong degeneration effects of the electron-hole plasma at cryogenic temperatures and takes the Fermi-Dirac statistics and nonlinear diffusion via a generalized Einstein relation fully into account [71]. For time-dependent simulations, we use an implicit Euler discretization and an adaptive time stepping method.

The discretized van Roosbroeck system is solved along with the QME (4) by a full Newton iteration using the electrostatic potential $\psi$, the quasi-Fermi energies $\mu_{c}, \mu_{v}$ and the density matrix elements $\langle k|\rho| l\rangle$ as independent variables. In order to obtain a system of ordinary differential equations, the QME is projected on the Hilbert space basis spanned by the multi-particle eigenstates of $H$ (see Appendix E).

The coupling terms $Q$ and $S_{n / p}$ given by Eq. $13 p$ and (14) introduce a non-local coupling of the van Roosbroeck system with the QME via the spatial profile function $w$. This has an impact on the sparsity pattern of the Jacobian of the discretized system, since the quantum system interacts in general with a large number of control volumes in its environment. Since the discretized spatial profile function $w_{K}=\left|\Omega_{K}\right|^{-1} \int_{\Omega_{K}} \mathrm{~d}^{3} r w(\mathbf{r})$ (where $\left|\Omega_{K}\right|$ is the volume of the $K$-th Voronoï cell), quickly decays, we discard small matrix elements below a chosen threshold. This preserves the quadratic convergence of Newton's iteration while the numerical effort is reduced.

Single-photon sources are typically operated at cryogenic temperatures, which causes serious convergence issues during the numerical solution of the van Roosbroeck 
system because of the strong depletion of minority carrier densities [71 73]. By using the temperature embedding method described in Ref. [71], the problem becomes tractable in the vicinity of flat band conditions.

\section{Device specification}

In the numerical simulations presented in the following, we consider the cylindrical GaAs-based p-i-n structure depicted in Fig. 3, where a single QD is placed on the symmetry axis within the center of the intrinsic zone. The total height of the device is $800 \mathrm{~nm}$, the intrinsic layer has a thickness of $200 \mathrm{~nm}$ and the doped layers both are $300 \mathrm{~nm}$ in height. The doping concentrations are $C=N_{D}=2 \times 10^{18} \mathrm{~cm}^{-3}$ and $C=-N_{A}=-10^{19} \mathrm{~cm}^{-3}$ in the $\mathrm{n}$ - and p-domain, respectively. The top radius of the mesa is $0.5 \mu \mathrm{m}$ and the total radius (at the bottom) is $2.5 \mu \mathrm{m}$. The bottom facet is assumed to consist of a highly reflective metal such that it simultaneously acts as an electric contact and a mirror leading to a directed emission in vertical direction. The ohmic contact on the top facet is assumed to consist of an optically transparent material, such that the structure forms a leaky cavity with a low $Q$ factor. The remaining facets are modeled by homogeneous Neumann boundary conditions. The wetting layer (WL) indicated in Fig. 3 is neglected in the simulation. The device is assumed to operate under cryogenic conditions at $T=50 \mathrm{~K}$.

The numerical simulation exploits the rotational symmetry of the device, such that the computational domain reduces to a $2 \mathrm{D}$ cross section with adapted cell volumes.

\section{Stationary operation}

The device operates as a p-i-n diode, which can be seen from the current-voltage curve shown in Fig. 5(c). At cryogenic temperatures the Fermi energy levels in the doped domains are very close to the band edges and therefore the diode's threshold voltage approximately equals the energy band gap of the material (around $1.52 \mathrm{~V}$ ). The population of the QD states $\langle k\rangle=\langle k|\rho| k\rangle$ can be controlled by the externally applied bias as shown in Fig. 5(a). Since the QD is located within the intrinsic zone of the device, it is most probably unoccupied in the low bias regime. When the applied bias approaches the diode's threshold voltage, the QD population turns into a non-equilibrium distribution: At first, due to the increased continuum carrier densities in the vicinity of the QD, the single-particle and excitonic states are populated. In particular, due to the lack of an radiative decay channel, the dark excitons $\left\langle D_{1 / 2}\right\rangle$ have a high occupation probability. Finally, beyond the threshold, the QD is quickly driven into saturation and the population is dominated by the biexciton state $\langle B\rangle$. Due to Coulomb enhancement and suppression, the population of neutral states is favored in the whole bias range. In particular,
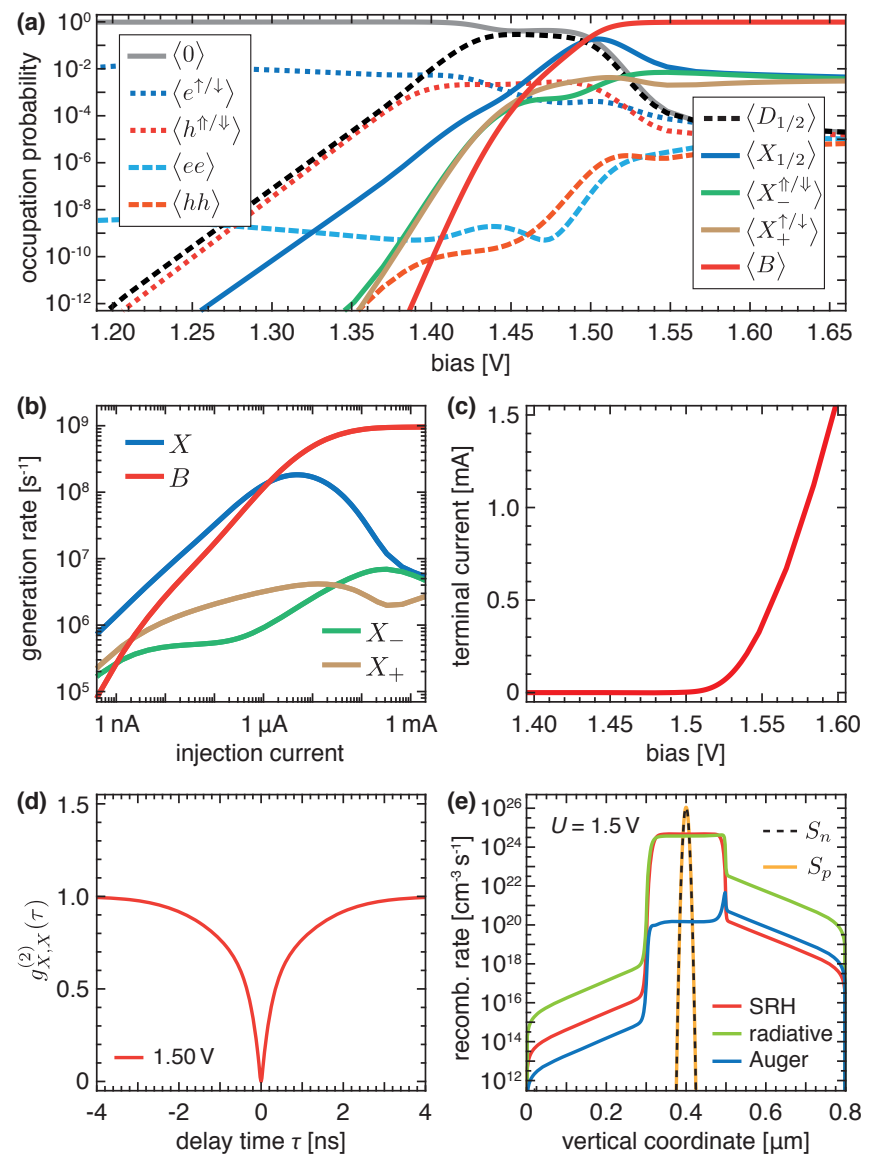

Figure 5. Numerical results at stationary injection. (a) Occupation of the QD states vs. applied bias. (b) Single-photon generation rates of the different emission lines vs. injection current. (c) Current-voltage curve of the diode. (d) Second order correlation function of the photons generated on the exciton line. (e) Comparison of recombination and capture rates of free carriers along the symmetry axis of the device. For the labeling of QD states we refer to the caption of Fig. 4.

Fig. 5(a) shows that the population of the doubly charged states $\langle e e\rangle$ and $\langle h h\rangle$ is strongly suppressed.

The single-photon generation rates of the different emission lines are given by

$$
\Gamma_{k}=\sum_{l} \mathcal{A}_{k, l}^{0} \gamma_{k \rightarrow l}^{0}\langle k\rangle
$$

Since the decay rates for all radiative processes are approximately equal, the occupation probabilities are directly proportional to the single-photon generation rates, which are depicted in Fig. 5(b). At low injection currents, the emission spectrum is dominated by photons generated via the decay of bright excitons. Close to the threshold voltage the bright exciton line reaches a maximum and then decreases while the intensity of the biexciton line grows until it finally saturates. In this regime, the capture rates exceed the radiative decay rates by several orders of magnitude. This simulation result agrees with experimental observations presented in Ref. [58. 
(a) excitation pulse

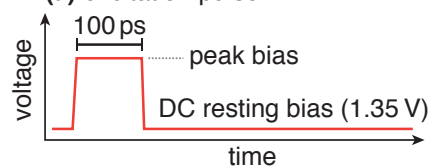

carrier density $\left[\mathrm{cm}^{-3}\right]$

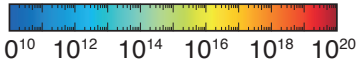

(b) holes

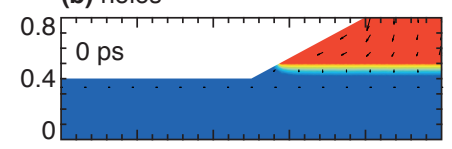

(c) electrons
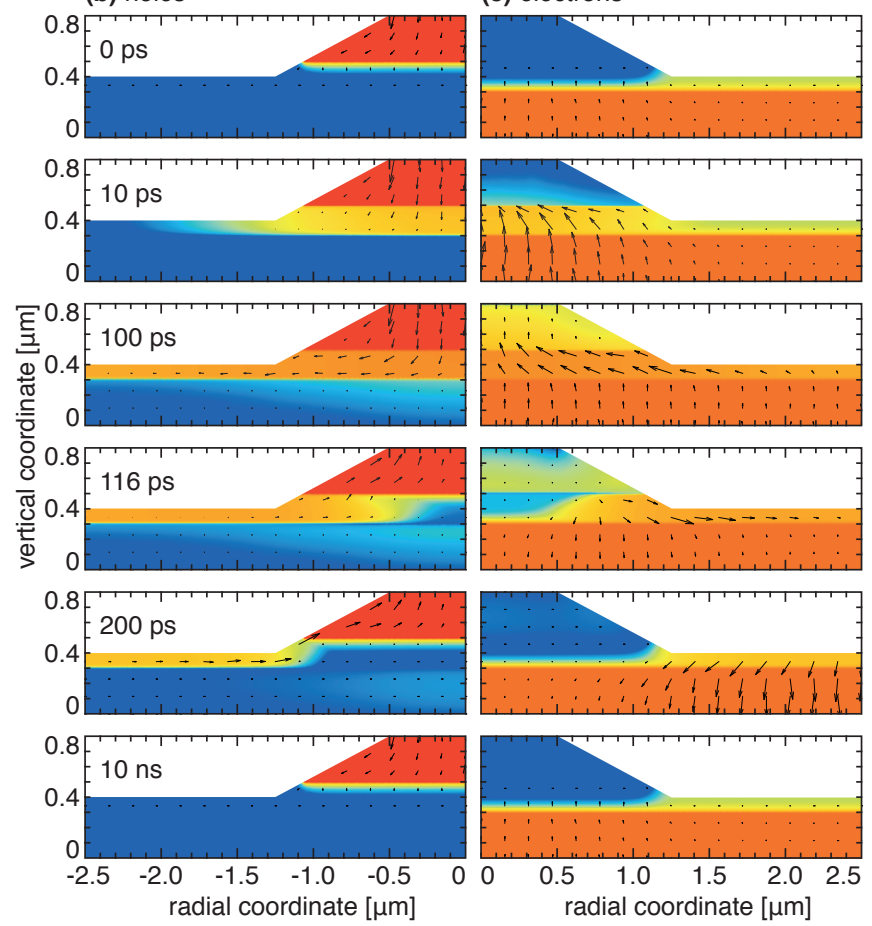

Figure 6. Carrier transport at pulsed excitation. (a) Illustration of the voltage ramp used in the simulations. (b, c) Snapshots of the carrier density distribution on a 2D crosssection at several instances of time. The carrier density is color-coded, the arrows indicate the current density vector field (arrows point into the direction of particle motion). The peak voltage in the simulation was set to $1.6 \mathrm{~V}$.

Another important figure of merit for single-photon emitters is the second order intensity correlation function of the generated photons

$$
g^{(2)}(\tau)=\frac{\left\langle a^{\dagger}(0) a^{\dagger}(\tau) a(\tau) a(0)\right\rangle}{\left\langle a^{\dagger}(0) a(0)\right\rangle^{2}},
$$

where the operator $a^{\dagger}(a)$ creates (annihilates) a photon and $\tau$ is a time delay. A value of $g^{(2)}(0)<0.5$ indicates the presence of a single-photon Fock state in the radiation field. In our model the decay of an optically active $\mathrm{QD}$ state is equivalent to the generation of a corresponding photon. Therefore, the electronic operators can be used to evaluate Eq. (30), cf. Ref. 65. For the bright exciton line, we identify the photon creation operator with the projector $a^{\dagger}=|0\rangle\left\langle X_{i}\right|$ (with $i=1$ or 2 ) and use the quantum regression theorem 28, to evaluate Eq. (30). The result is presented in Fig. $5(\mathrm{~d})$ and recovers the characteristic dip around $\tau=0$ for high-quality single-photon sources [13, 58]. Since the present model
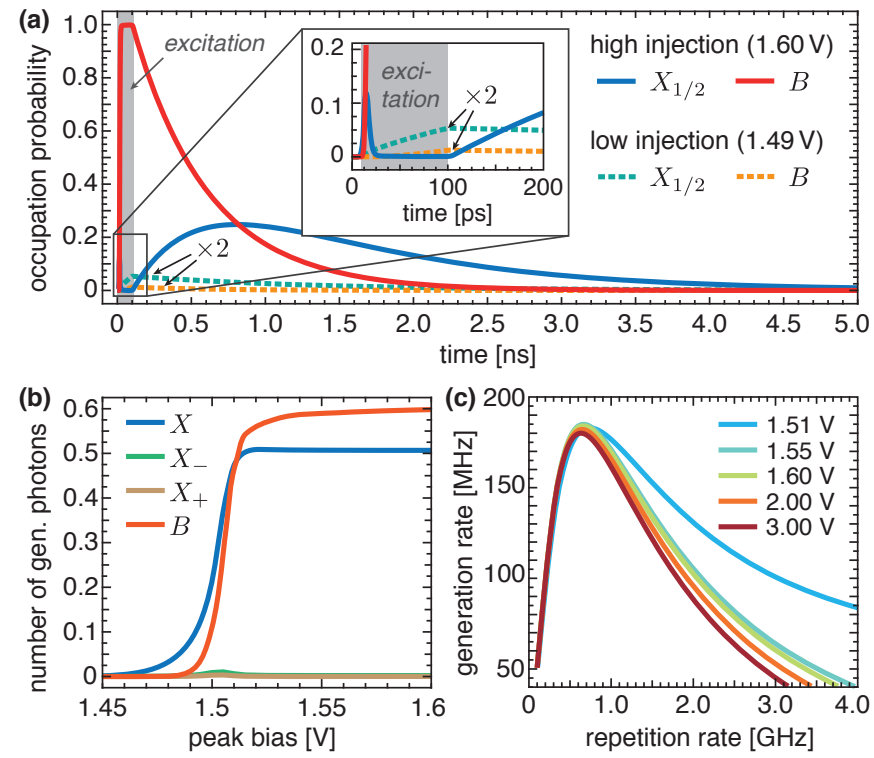

Figure 7. QD occupation and single-photon generation under pulsed excitation. (a) Comparison of the transient exciton and biexciton occupation probabilities in the low and high injection case. In the low injection case the occupation probabilities have been multiplied by a factor 2 for better visibility. (b) Number of generated photons per pulse on the different emission lines for different values of the peak bias. (c) Single-photon generation rate on the bright exciton line vs. repetition frequency of the time-periodic excitation pulse (for different values of the peak bias).

assumes an ideal quantum emitter and an instantaneous extraction of the generated photons from the cavity, the value of $g^{(2)}(0)$ is exactly zero. For a refined description at this stage, a coherent light-matter interaction must be included in the Hamiltonian and $\mathcal{D}_{0}$ has to be extended by a photon outcoupling mechanism.

Finally, in Fig. 5(e) we show the recombination rate $R$ of the continuum carriers and the capture rates $S_{n / p}$ along the vertical (symmetry) axis of the device. Close to the threshold voltage, the transition of carriers into the QD imposes the dominant loss mechanism of continuum carriers in the vicinity of the QD.

\section{E. Pulsed operation}

For many applications, the generation of single photons at certain instances of time is required. Electrically driven QD-based single-photon sources offer an easy off-resonant excitation scheme [4, 65], where the QD is excited by short voltage pulses. This process shall be simulated in the following, where we apply rectangular voltage pulses with a fixed duration of $100 \mathrm{ps}$ superimposed on a DC bias of $1.35 \mathrm{~V}$ as illustrated in Fig. 6(a). We investigate the impact of the pulse repetition time and the peak bias, which are the key external control parameters. The results of a numerical carrier transport 
simulation for a single pulse with a peak voltage of $1.6 \mathrm{~V}$ are shown in Fig. 6(b, c). Due to the high carrier mobilities at low temperatures (cf. Appendix F), the carriers quickly spread out within the device such that the intrinsic zone is highly populated at the end of the excitation pulse (100 ps). Subsequently, when the applied voltage is switched back to the resting DC bias, the carriers are quickly withdrawn from the intrinsic zone. In the snapshots taken at $116 \mathrm{ps}$ and $200 \mathrm{ps}$ we observe that in particular the vicinity of the QD (which is located on the center of the symmetry axis at $0.4 \mu \mathrm{m}$, cf. Fig. 3 ) is depleted first. Moreover, a conducting channel underneath the insulating region is formed. The plot at $10 \mathrm{~ns}$ shows the stationary state reached after a long time.

The impact of the voltage pulse on the occupation of the QD is shown in Fig. 77(a). In the case of an excitation with a peak voltage of $1.6 \mathrm{~V}$ (high injection), one first observes a fast occupation of the biexciton state which subsequently decays radiatively. Via the so-called biexciton-cascade, the bright exciton states are populated in the following. Comparing the time scales of the carrier transport with the life times of the bright QD states, see Fig. 6(b, c) and Fig. 7(a), it is apparent that the decay of the bright exciton happens a long time after the continuum carriers have left the vicinity of the QD. This separation of time scales is of particular importance for the generation of indistinguishable photons [59, since fluctuations of the carrier density in the vicinity of the emitter might shift the generated photon's energy.

Next, we study the impact of the peak bias value. Figure 7 (b) shows the number of generated photons for different peak voltages after $10 \mathrm{~ns}$. The number of generated photons on line $k$ until time $t$ is obtained from

$$
N_{k}(t)=\int_{0}^{t} \mathrm{~d} t^{\prime} \Gamma_{k}\left(t^{\prime}\right),
$$

using the single-photon generation rate defined in Eq. 29). The plot clearly reveals the existence of two regimes: A subthreshold (low injection) regime, where the peak voltage is insufficient for the excitation of the QD (cf. Fig. 77(a)), and a high injection regime where the biexciton-cascade can be observed practically after each pulse. For the exciton-photons, this implies a generation efficiency of around $50 \%$ for both polarizations. The generation efficiency of the two differently polarized photons on the biexciton-line is a little higher than $50 \%$, due to additional recombination during the excitation period, see Fig. 7(a, b).

Finally, we investigate the optimal repetition frequency of the excitation cycle for the generation of single excitonphotons. The optimal repetition frequency $f^{*}=1 / t^{*}$ maximizes the number of generated photons per time:

$$
\bar{\Gamma}_{X}\left(t^{*}\right)=\frac{N_{X}\left(t^{*}\right)}{t^{*}}=\frac{1}{t^{*}} \int_{0}^{t^{*}} \mathrm{~d} t^{\prime} \Gamma_{X}\left(t^{\prime}\right) \rightarrow \max .
$$

Figure 7(c) shows a clear maximum at a pulse repetition rate of $f^{*} \approx 650 \mathrm{MHz}\left(t^{*} \approx 1.5 \mathrm{~ns}\right)$, which corresponds to a maximum single-photon generation rate of

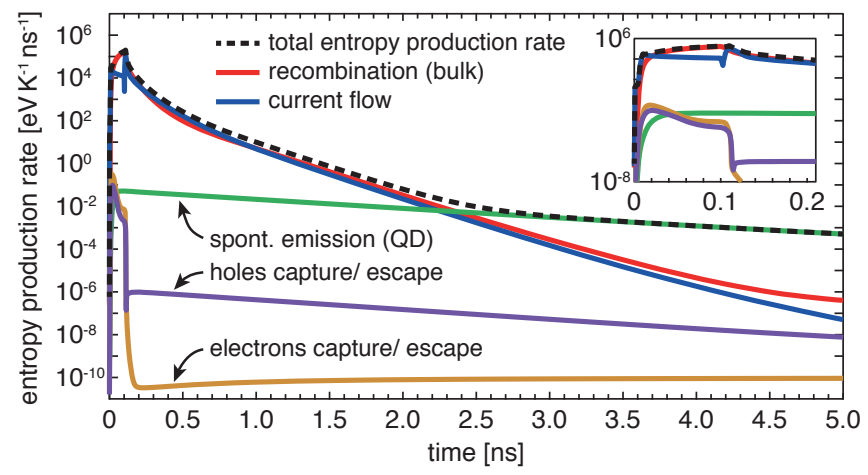

Figure 8. Entropy production rate during pulsed excitation (peak bias $1.6 \mathrm{~V}$ ). The plot shows the five contributions arising from the individual lines in Eq. 24) and the total entropy production rate. The inset is a zoom on the first $200 \mathrm{ps}$.

$\bar{\Gamma}_{X}\left(t^{*}\right) \approx 185 \mathrm{MHz}$. Even though in this optimal case the photon generation efficiency per pulse shrinks to $28 \%$, the high repetition frequency leads to an enhanced overall performance. Moreover, Fig. 7(c) indicates that this result is practically independent of the peak voltage. In order to obtain the actual emission rate, the generation rate must be multiplied with the extraction efficiency [74].

We conclude this section with a consideration of the entropy production during an excitation cycle, which is depicted in Fig. 8 The plot shows, that during the first $2 \mathrm{~ns}$ the entropy production rate is clearly governed by the contributions arising from the macroscopic system, whereas at later times the slow decay of the QD-exciton becomes dominant. The numerical result is in agreement with the theory presented in Sec. IIID, which predicts a positive entropy production rate at all times.

\section{DISCUSSION AND OUTLOOK}

The electrically driven single-photon source considered in the previous section is a realistic application that fits into the framework of the new model system (11)-(4) introduced in this paper. This example is a proof of concept that shows the computational tractability of our approach regarding its potential for applications in numerical semiconductor device simulation. As demonstrated in Sec. IV] the hybrid approach enables a comprehensive analysis of QD-based devices even in the case of complex, multi-dimensional device geometries as well as the investigation of transient processes.

If the feedback of the quantum system on its classical environment is weak, i.e. if the capture rates $S_{n / p}$ are small compared to the recombination rate $R$, simplified approaches can be considered. A first option is to merely consider the semi-classical transport while neglecting the quantum system as carried out e.g. in Ref. [24]. In this case, however, the model gives no access on the quantum optical figures of merit, of course. A second option is to treat the quantum master equation alone by choosing 
an appropriate parametrization of the transition rates in the dissipation superoperators as done e.g. in Ref. 65. However, in electrically driven devices, the carrier densities, the electric field and the current densities, which usually drive the transition rates, strongly depend on the applied voltage and can vary over many orders of magnitude. In general, their detailed behavior is not apriori known and requires full device simulation since the evolution of these quantities is determined by specific design parameters such as the device geometry, doping profiles, heterostructures etc. In conclusion, the hybrid modeling approach described in this paper goes beyond existing ones.

In the case of weak feedback, the coupling of both subsystems in the hybrid model becomes effectively unidirectional. This means that the dynamics of the quantum system is slaved by the evolution of its classical environment, which can be exploited to reduce the computational effort in a two-step method: First, the transport simulation is carried out whilst omitting coupling terms to the quantum system. In a second step, the solution of the classical system is used to determine the timedependent dissipators that drive the evolution of the open quantum system. Hence, the quantum master equation is solved in a "post-processing" step, which finally gives access to the quantum optical figures of merit. Via the explicit dependency of the microscopic transition rates on the state of the classical environment (spatially averaged macroscopic potentials), the hybrid model provides a consistent link between the two steps of the unidirectionally coupled simulation approach. Nevertheless, even in the case of weak feedback, where one-way coupled approaches are admissible, the fully coupled hybrid model allows to assess the approximation errors. Thereby it helps to justify simplified simulation approaches.

The application considered in Sec. [V] is an example for a quantum system with a weak feedback on the classical environment, which in principle would allow for the one-way method outlined above. The essential reason for this is the slow radiative decay in comparison to the fast electronic processes, which keeps the capture rates $S_{n / p}$ small once the QD is occupied. However, this is not always the case. For example, in electrically driven QD nanolasers, where the QD is placed inside a resonant cavity, the Purcell-enhanced light-matter interaction strongly decreases the radiative carrier lifetimes [8]. As a consequence, the capture rates $S_{n / p}$ are expected to increase by some orders of magnitude such that the quantum system significantly couples back to its classical environment and contributes to current guiding. We suspect that in this case the predictions of the hybrid model differ clearly from a decoupled treatment.

An interesting extension of the system (1)-(4) concerns reservoirs with different temperatures, as frequently studied in quantum thermodynamics [39, 43]. We are confident that it is possible to achieve a thermodynamically consistent coupling of the quantum master equation (4) with energy transport models [44] or other trans- port models taking higher moments of the semi-classical Boltzmann equation [75] into account. The latter extend the isothermal van Roosbroeck system by one or multiple heat flow equations that determine the spatial temperature distribution of the crystal lattice and the continuum carriers. The construction of the corresponding hybrid system should be analogous to the case considered in this paper. The essential difference is that the coupling of both subsystems involves spatially averaged thermodynamic forces instead of chemical potentials, e.g. $\left\langle\mu_{c}\right\rangle_{w} \rightarrow\left\langle\mu_{c} / T\right\rangle_{w}$ etc. What might be interesting in the non-isothermal case is the impact of the quantumclassical interactions on the heat generation.

\section{SUMMARY}

Nowadays, quantum optical technologies are on their way from the lab to real world applications. To advance this development, device engineers will need simulation tools, which combine classical device physics with models from cavity quantum electrodynamics. As a step on this route, we have presented a new modeling approach for the simulation of single and few quantum dot devices.

By connecting semi-classical carrier transport theory with a quantum master equation in Lindlad form, our approach has lead to a hybrid quantum-classical system, that allows for a comprehensive description of electrically driven quantum dot devices on multiple scales: It enables the computation of the spatially resolved carrier transport together with the calculation of quantum optical figures of merit (e.g. photon generation rates, higher order correlation functions) in realistic semiconductor structures in a unified way. This has been demonstrated by numerical simulations of an electrical single-photon source based on a single quantum dot. We have presented a thorough theoretical analysis of the approach and showed that it guarantees the conservation of charge and the consistency with the thermodynamic equilibrium. Finally, we have proven that our hybrid quantum-classical system obeys the second law of thermodynamics.

We believe that our approach serves as a blueprint for the simulation of further quantum dot based photonic devices, in particular nanolasers.

\section{ACKNOWLEDGMENTS}

The work of M.K. has been support by the Deutsche Forschungsgemeinschaft (DFG) within the collaborative research center 787 Semiconductor Nanophotonics under grant B4. M.M. was supported by the ERC via AdG 267802 AnaMultiScale. The authors acknowledge valuable discussions with H.-J. Wünsche, U. Bandelow, D. Peschka and A. Mielke. The authors are grateful to the reviewer for the detailed and helpful comments. 


\section{Appendix A: Boundary conditions}

We assume a decomposition of the domain boundary

$$
\partial \Omega=\left(\bigcup_{i} \Gamma_{i}\right) \cup \partial \Omega_{N}
$$

into several ohmic contacts and artificial boundaries of the device [32]. On the artificial boundaries $\partial \Omega_{N}$, we assume homogeneous Neumann conditions

$$
\mathbf{n} \cdot \nabla \psi=0, \quad \mathbf{n} \cdot \nabla \mu_{c}=0, \quad \mathbf{n} \cdot \nabla \mu_{v}=0,
$$

where $\mathbf{n}$ denotes the outer normal vector. The ohmic contacts are modeled by Dirichlet boundary conditions

$$
\psi=\psi_{\mathrm{eq}}+U_{\mathrm{appl}, i}, \quad \mu_{c}=\mu_{i}, \quad \mu_{v}=\mu_{i},
$$

on $\Gamma_{i}$, where $U_{\mathrm{appl}, i}$ represents the applied voltage at the $i$-th ohmic contact and $\mu_{i}=\mu_{\text {eq }}-q U_{\text {appl }, i}$. The value of the built-in potential $\psi_{\text {eq }}$ is obtained from the local charge neutrality condition at the ohmic boundaries and zeros bias conditions $\left(\mu_{i} \equiv \mu_{\text {eq }} \forall i\right)[67$.

\section{Appendix B: Electrostatic field energy}

Following [45], we split the electrostatic potential

$$
\psi=\psi_{\text {int }}+\psi_{\text {ext }}
$$

into an internal field $\psi_{\text {int }}$ generated by the internal charge density and an external field $\psi_{\text {ext }}$, which arises from the built-in doping profile and the applied voltages. Consequently, the Poisson problem (1) is decomposed into

$$
\begin{aligned}
& -\nabla \cdot \varepsilon \nabla \psi_{\mathrm{int}}=q \rho_{\mathrm{int}}, \\
& -\nabla \cdot \varepsilon \nabla \psi_{\mathrm{ext}}=q C,
\end{aligned}
$$

such that the internal field $\psi_{\text {int }}=\psi_{\text {int }}\left(\rho_{\text {int }}\right)$ can be written as a functional of the total internal carrier density

$$
\rho_{\mathrm{int}}=p-n+Q(\rho) .
$$

On the domain boundaries it holds

$$
\begin{aligned}
& \mathbf{n} \cdot \varepsilon \nabla \psi_{\text {int }}=0 \quad \text { on } \partial \Omega_{N}, \\
& \psi_{\text {int }}=0 \quad \text { on } \Gamma_{i},
\end{aligned}
$$

and

$$
\begin{aligned}
\mathbf{n} \cdot \varepsilon \nabla \psi_{\mathrm{ext}} & =0 & & \text { on } \partial \Omega_{N}, \\
\psi_{\mathrm{ext}} & =\psi_{\mathrm{eq}}+U_{\mathrm{appl}, i} & & \text { on } \Gamma_{i} .
\end{aligned}
$$

A variation of the internal carrier density $\rho_{\text {int }} \rightarrow \rho_{\text {int }}+$ $a \delta \rho(0<a \ll 1$ is a small parameter $)$ in the interior of the domain yields a variation of the electrostatic field $\delta \psi$ according to

$$
-\nabla \cdot \varepsilon \nabla \delta \psi=q \delta \rho \quad \text { on } \Omega
$$

with the same boundary conditions for $\delta \psi$ as for $\psi_{\text {int }}$ stated above. The variation of the internal energy given by Eq. 20 leads to

$$
\begin{aligned}
U_{\psi}\left(\rho_{\text {int }}+a \delta \rho\right)= & U_{\psi}\left(\rho_{\text {int }}\right) \\
& +a \int_{\Omega} \mathrm{d}^{3} r \varepsilon \nabla \psi_{\text {int }}\left(\rho_{\text {int }}\right) \cdot \nabla \delta \psi \\
& +a q \int_{\Omega} \mathrm{d}^{3} r \delta \rho \psi_{\text {ext }}+\mathcal{O}\left(a^{2}\right) .
\end{aligned}
$$

Finally, using the identity

$$
\int_{\Omega} \mathrm{d}^{3} r \varepsilon \nabla \psi_{\text {int }}\left(\rho_{\text {int }}\right) \cdot \nabla \delta \psi=q \int_{\Omega} \mathrm{d}^{3} r \psi_{\text {int }}\left(\rho_{\text {int }}\right) \delta \rho,
$$

one obtains the Gâteaux-derivative

$$
\begin{aligned}
& \lim _{a \rightarrow 0} \frac{U_{\psi}\left(\rho_{\text {int }}+a \delta \rho\right)-U_{\psi}\left(\rho_{\text {int }}\right)}{a}= \\
&=q \int_{\Omega} \mathrm{d}^{3} r\left(\psi_{\text {int }}\left(\rho_{\text {int }}\right)+\psi_{\text {ext }}\right) \delta \rho .
\end{aligned}
$$

The central feature of the field's internal energy expression Eq. 200 is 44, 45]

$$
\frac{\delta U_{\psi}}{\delta \rho}=q \psi
$$

\section{Appendix C: Entropy production rate}

This section gives some details on the derivation of the expression (24) for the entropy production rate. Starting from Eq. (15), one obtains by using Eq. (17) and (18) the entropy production rate as

$$
\begin{aligned}
\frac{\mathrm{d} S_{\mathrm{tot}}}{\mathrm{d} t}= & -\frac{1}{T} \int_{\Omega} \mathrm{d}^{3} r\left(\frac{\partial u_{\mathrm{cl}}(n, p)}{\partial t}-T \frac{\partial s_{\mathrm{cl}}(n, p)}{\partial t}\right) \\
& -\frac{1}{T} \operatorname{tr}\left(H \mathcal{L}\left(\rho ; \chi_{w}\right)\right)-k_{B} \operatorname{tr}\left(\log (\rho) \mathcal{L}\left(\rho ; \chi_{w}\right)\right) \\
& -\frac{1}{T} \frac{\mathrm{d} U_{\psi}}{\mathrm{d} t}+\sum_{i \geq 1} \frac{\mu_{i}}{q T} \int_{\Gamma_{i}} \mathrm{~d} \mathbf{A} \cdot\left(\mathbf{j}_{n}+\mathbf{j}_{p}\right) .
\end{aligned}
$$

Taking the partial time derivatives, using the state equations (5), Eq. (B1) and

$$
\frac{\mathrm{d} U_{\psi}}{\mathrm{d} t}=\int_{\Omega} \mathrm{d}^{3} r q \psi \frac{\partial(p-n+Q(\rho))}{\partial t},
$$

we arrive at

$$
\begin{aligned}
\frac{\mathrm{d} S_{\text {tot }}}{\mathrm{d} t}= & -\frac{1}{T} \int_{\Omega} \mathrm{d}^{3} r\left(\mu_{c} \frac{\partial n}{\partial t}-\mu_{v} \frac{\partial p}{\partial t}\right) \\
& -\frac{1}{T} \operatorname{tr}\left(H \mathcal{L}\left(\rho ; \chi_{w}\right)\right)-k_{B} \operatorname{tr}\left(\log (\rho) \mathcal{L}\left(\rho ; \chi_{w}\right)\right) \\
& +\frac{q}{T}\langle\psi\rangle_{w} \operatorname{tr}\left(N \mathcal{L}\left(\rho ; \chi_{w}\right)\right) \\
& +\sum_{i \geq 1} \frac{\mu_{i}}{q T} \int_{\Gamma_{i}} \mathrm{~d} \mathbf{A} \cdot\left(\mathbf{j}_{n}+\mathbf{j}_{p}\right)
\end{aligned}
$$


where we have explicitly used Eq. 13. for the charge density of the quantum system. For different $Q(\rho)$ and multiple QDs, the calculation follows the same lines. With the help of the carrier transport equations (2)-(3), the macroscopic capture rates (14), partial integration and the boundary conditions given in Appendix $\mathrm{A}$ this is

$$
\begin{aligned}
\frac{\mathrm{d} S_{\mathrm{tot}}}{\mathrm{d} t}= & \frac{1}{T} \int_{\Omega} \mathrm{d}^{3} r\left(\mu_{c}-\mu_{v}\right) R \\
& +\frac{1}{q T} \int_{\Omega} \mathrm{d}^{3} r\left(\mathbf{j}_{n} \cdot \nabla \mu_{c}+\mathbf{j}_{p} \cdot \nabla \mu_{v}\right) \\
& +\frac{1}{T}\left\langle\mu_{c}\right\rangle_{w} \operatorname{tr}\left(N \mathcal{D}_{e}\left(\rho ; \chi_{w}\right)\right) \\
& +\frac{1}{T}\left\langle\mu_{v}\right\rangle_{w} \operatorname{tr}\left(N \mathcal{D}_{h}\left(\rho ; \chi_{w}\right)\right) \\
& -\frac{1}{T} \operatorname{tr}\left(H \mathcal{D}\left(\rho ; \chi_{w}\right)\right)-k_{B} \operatorname{tr}\left(\log (\rho) \mathcal{D}\left(\rho ; \chi_{w}\right)\right) \\
& +\frac{q}{T}\langle\psi\rangle_{w} \operatorname{tr}\left(N \mathcal{D}\left(\rho ; \chi_{w}\right)\right) .
\end{aligned}
$$

In the above expression, the surface integrals have canceled out. Using Eqns. 111- 12, one arrives at Eq. 24.

\section{Appendix D: Second law of thermodynamics}

In this section we proof the non-negativity of the entropy production rate (24) of the hybrid system (1)-(4). First, we introduce the (auxiliary) density matrices

$$
\begin{aligned}
\rho_{0}^{*} & =\frac{1}{Z_{0}^{*}} e^{-\beta H}, \\
\rho_{e}^{*}\left(\chi_{w}\right) & =\frac{1}{Z_{e}^{*}} e^{-\beta\left(H-\mu_{c}^{\mathrm{eff}}\left(\chi_{w}\right) N\right)}, \\
\rho_{h}^{*}\left(\chi_{w}\right) & =\frac{1}{Z_{h}^{*}} e^{-\beta\left(H-\mu_{v}^{\mathrm{eff}}\left(\chi_{w}\right) N\right)}
\end{aligned}
$$

with $\mu_{c / v}^{\mathrm{eff}}\left(\chi_{w}\right)=\left\langle\mu_{c / v}\right\rangle_{w}+q\langle\psi\rangle_{w}$. Using Eq. 233 it can be shown by direct calculation that $\mathcal{D}_{\nu}\left(\rho_{\nu}^{*}\left(\chi_{w}\right) ; \chi_{w}\right)=0$, $\nu \in\{0, e, h\}$, for the dissipators given in Eq. (11). Then, Spohn's inequaility [53] states that

$$
\operatorname{tr}\left(\left(\log \rho_{\nu}^{*}\left(\chi_{w}\right)-\log \rho\right) \mathcal{D}_{\nu}\left(\rho ; \chi_{w}\right)\right) \geq 0
$$

for $\nu \in\{0, e, h\}$.

The entropy production rate Eq. 24 can be written in the form

$$
\begin{aligned}
\frac{\mathrm{d} S_{\mathrm{tot}}}{\mathrm{d} t}= & k_{B} \int_{\Omega} \mathrm{d}^{3} r \beta\left(\mu_{c}-\mu_{v}\right)\left(1-e^{-\beta\left(\mu_{c}-\mu_{v}\right)}\right) \sum_{j} r_{j} \\
& +\frac{1}{q^{2} T} \int_{\Omega} \mathrm{d}^{3} r\left(\sigma_{n}\left|\nabla \mu_{c}\right|^{2}+\sigma_{p}\left|\nabla \mu_{v}\right|^{2}\right) \\
& +k_{B} \operatorname{tr}\left(\left(\log \rho_{0}^{*}-\log \rho\right) \mathcal{D}_{0}\left(\rho ; \chi_{w}\right)\right) \\
& +k_{B} \operatorname{tr}\left(\left(\log \rho_{e}^{*}\left(\chi_{w}\right)-\log \rho\right) \mathcal{D}_{e}\left(\rho ; \chi_{w}\right)\right) \\
& +k_{B} \operatorname{tr}\left(\left(\log \rho_{h}^{*}\left(\chi_{w}\right)-\log \rho\right) \mathcal{D}_{h}\left(\rho ; \chi_{w}\right)\right)
\end{aligned}
$$

where we have used Eqns. (6), (D1), the trace conservation property of the dissipator and a recombination rate of the form (F1). Here, $j$ labels the recombination channels and the functions $r_{j}=r_{j}(n, p, \psi)$ are non-negative by construction (cf. Appendix F 1). Using the inequalities (D2) and $x\left(1-e^{-x}\right) \geq 0 \forall x \in \mathbb{R}$, it is easy to see that each line of Eq. (D3) is non-negative.

\section{Appendix E: Projection on eigenstates}

In order to obtain a system of ODEs from Eq. (4), it must be projected on a basis of the quantum system's Hilbert space. We use the eigenbasis of the Hamiltonian $H$, for which we assume the spectral representation

$$
H=\sum_{k} \varepsilon_{k}\left|\varphi_{k}\right\rangle\left\langle\varphi_{k}\right| .
$$

For the sake of simplicity, we consider the energy spectrum $\left\{\varepsilon_{k}\right\}$ to be non-degenerate here. Moreover, the Lamb-Shift contribution to $H$ is neglected. Then, the jump operators are projectors between energy eigenstates $A_{\alpha} \rightarrow A_{i, j}=\left|\varphi_{i}\right\rangle\left\langle\varphi_{j}\right|$. The equations of motion for the diagonal elements of the density matrix are obtained as

$$
\partial_{t}\left\langle\varphi_{k}|\rho| \varphi_{k}\right\rangle=\sum_{j}\left(\mathcal{M}_{k, j}\left\langle\varphi_{j}|\rho| \varphi_{j}\right\rangle-\mathcal{M}_{j, k}\left\langle\varphi_{k}|\rho| \varphi_{k}\right\rangle\right),
$$

whereas the off-diagonal elements $k \neq l$ obey

$$
\begin{aligned}
\partial_{t}\left\langle\varphi_{k}|\rho| \varphi_{l}\right\rangle= & -\frac{i}{\hbar}\left(\varepsilon_{k}-\varepsilon_{l}\right)\left\langle\varphi_{k}|\rho| \varphi_{l}\right\rangle \\
& -\frac{1}{2} \sum_{j}\left(\mathcal{M}_{j, k}+\mathcal{M}_{j, l}\right)\left\langle\varphi_{k}|\rho| \varphi_{l}\right\rangle
\end{aligned}
$$

with the (non-negative) transition rate matrix elements

$$
\begin{aligned}
\mathcal{M}_{i, j} & =\gamma_{i, j}+\hat{\gamma}_{j, i} \\
& =\gamma_{i, j}\left(1+e^{-\beta\left(\varepsilon_{j}-\varepsilon_{i}-\left(\left\langle\mu_{i, j}\right\rangle_{w}+q\langle\psi\rangle_{w}\right) \ell_{i, j}\right)}\right) \geq 0 .
\end{aligned}
$$

Obviously, in the case of non-degenerate energy spectra the diagonal elements decouple from the off-diagonal elements. The off-diagonal elements are fully decoupled each and show damped oscillations (dephasing). This has important implications on the complexity of the numerical simulations: Starting from the thermodynamic equilibrium state, where only diagonal elements of the density matrix are occupied, the dynamics never excite any off-diagonal elements (in the energy eigenbasis representation). Hence, the off-diagonal elements can be omitted from the simulation. Thereby the number of degrees of freedom of the quantum system grows only with $N$ instead of $N^{2}$, where $N$ is the dimension of the (possibly truncated) Hilbert space. However, if the spectrum of $H$ is degenerate, this feature is lost in general and one has to account for degenerate eigenstate coherences (i.e. off-diagonal elements contribute to the dynamics) [37. 


\section{Appendix F: Parameters and auxiliary models}

This section lists auxiliary models and parameters used in the numerical simulations presented in Sec. IV.

\section{Van Roosbroeck system}

We use GaAs parameters at $T=50 \mathrm{~K}$. The effective masses are $m_{e}^{*}=0.068 m_{0}, m_{h}^{*}=0.503 m_{0}$, where $m_{0}$ denotes the (free) electron mass and the band edge energies are taken as $E_{v}=0 \mathrm{eV}$ and $E_{c}=1.516 \mathrm{eV}$. The (static) relative permittivity is set to $\varepsilon_{r}=12.9$, the LO-phonon energy is $\hbar \omega_{\mathrm{LO}}=36.5 \mathrm{meV}$ and the refractive index is $n_{r}=3.55$ (around $950 \mathrm{~nm}$ ). The (net-)recombination rate in Eqns. (2), (3) is modeled as [32, 67]

$$
\begin{aligned}
R & =R_{\mathrm{SRH}}+R_{\mathrm{sp}}+R_{\mathrm{Au}} \\
& =\left(1-e^{-\beta\left(\mu_{c}-\mu_{v}\right)}\right) \sum_{j} r_{j}(n, p, \psi)
\end{aligned}
$$

where $j \in\{\mathrm{SRH}, \mathrm{sp}, \mathrm{Au}\}$ labels the different channels and

$$
\begin{aligned}
r_{\mathrm{SRH}} & =\frac{n p}{\tau_{p}\left(n+n_{d}\right)+\tau_{n}\left(p+p_{d}\right)} \\
r_{\mathrm{sp}} & =B n p \\
r_{\mathrm{Au}} & =\left(C_{\mathrm{Au}}^{n} n+C_{\mathrm{Au}}^{p} p\right) n p
\end{aligned}
$$

with $n_{d}=n e^{\beta\left(E_{T}-q \psi-\mu_{c}\right)}, p_{d}=p e^{-\beta\left(E_{T}-q \psi-\mu_{v}\right)}$. The recombination rates $r_{j}(n, p, \psi)$ of the individual channels are non-negative by construction. The non-radiative life times are sensitive to the impurity concentration and modeled via $\tau_{n / p}=\tau_{n / p, 0} /\left(1+\left(|C| / C_{\text {ref }}\right)^{\gamma_{\mathrm{SRH}}}\right)$ with $\tau_{n, 0}=\tau_{p, 0}=10 \mathrm{~ns}, \gamma_{\mathrm{SRH}}=1.72$ and $C_{\mathrm{ref}}=9 \times 10^{17} \mathrm{~cm}^{-3}$ [76]. The trap energy level $E_{T}$ is assumed to be in the center of the energy gap. The radiative recombination coefficient is taken as $B=1.06 \times 10^{-8} \mathrm{~cm}^{-3} \mathrm{~s}^{-1}$ and the Auger recombination coefficients are set to $C_{\mathrm{Au}}^{n}=$ $6 \times 10^{-30} \mathrm{~cm}^{-6} \mathrm{~s}^{-1}, C_{\mathrm{Au}}^{p}=1.6 \times 10^{-29} \mathrm{~cm}^{-6} \mathrm{~s}^{-1}[76]$. The carrier mobilities $M_{n / p}$ are taken from the model given in Ref. [77, which is reported to hold down to $T=50 \mathrm{~K}$. Despite the low temperatures, we assume complete ionization due to the metal-insulator transition at heavy doping 78 .

\section{Open quantum system}

The eigenenergies of the Hamiltonian (25) are obtained from the parabolic/step-like confinement potential (relative to the respective continuum band edge) $U_{\lambda}(r, z)=$ $-U_{0}^{\lambda} \Theta(h / 2-|z|)+\frac{1}{2} m_{\lambda}^{*} \omega_{\lambda, 0}^{2} r^{2}, \lambda \in\{e, h\}$, by solving the stationary Schrödinger equation at flat band conditions [48, 62. The parameters for the InGaAs-QD are taken as $U_{e}=350 \mathrm{meV}, U_{h}=170 \mathrm{meV}, m_{e}^{*}=0.067 m_{0}, m_{h}^{*}=$ $0.15 m_{0}$ [48 and $\hbar \omega_{e, 0}=45.5 \mathrm{meV}, \hbar \omega_{h, 0}=20.3 \mathrm{meV}$. The QD height is assumed as $h=3 \mathrm{~nm}$. For the computation of the Coulomb matrix elements we set the background dielectric permittivity to $\varepsilon_{r}=12.5$ [48].

With the parameters above, the QD conduction band ground state $\varepsilon_{c}$ is found at $137.7 \mathrm{meV}$ below the continuum band edge and the QD valence band ground state $\varepsilon_{v}$ is $44.7 \mathrm{meV}$ above the valence band edge. The Coulomb matrix elements are obtained as $V_{c, c}=23.2 \mathrm{meV}, V_{v, v}=$ $24.5 \mathrm{meV}$ and $V_{c, v}=23.7 \mathrm{meV}$. The interband dipole moment is assumed as $d_{c, v}=q \times 0.6 \mathrm{~nm}$ and the Purcell factor is set to $P_{i, f}=1.8$ for all allowed optical transitions. The emission energies are obtained around $1.31 \mathrm{eV}$ with radiative life times of approximately $1 \mathrm{~ns}$ according to Eq. 27). The fitting parameters in the carrier scattering rates are set to $\tau_{\mathrm{LO}}^{e}=\tau_{\mathrm{LO}}^{h}=10 \mathrm{ps}, a_{\mathrm{LO}}^{e}=25 \mathrm{meV}$, $a_{\mathrm{LO}}^{h}=7 \mathrm{meV}, \tau_{\mathrm{Au}}^{\lambda, \lambda^{\prime}}=1 \mathrm{ps}, \gamma_{\mathrm{Au}}^{\lambda, \lambda^{\prime}}=0.7$ (for all $\lambda, \lambda^{\prime} \in$ $\{e, h\}), n_{\mathrm{Au}}^{\text {crit }}=1 \times 10^{19} \mathrm{~cm}^{-3}$ and $p_{\mathrm{Au}}^{\text {crit }}=5 \times 10^{18} \mathrm{~cm}^{-3}$.
[1] D. Bimberg, M. Grundmann, and N. N. Ledentsov, Quantum Dot Heterostructures (John Wiley \& Sons, 1999).

[2] P. Michler, ed., Single Quantum Dots, Topics in Applied Physics, Vol. 90 (Springer, Berlin, Heidelberg, 2003).

[3] P. Bhattacharya and Z. Mi, Proc. IEEE 95, 1723 (2007).

[4] P. Michler, ed., Single Semiconductor Quantum Dots (Springer, Berlin, Heidelberg, 2009).

[5] D. Bimberg and U. W. Pohl, Materials Today 14, 388 (2011)

[6] S. Noda, Science 314, 260 (2006)

[7] C. Gies, M. Florian, P. Gartner, and F. Jahnke, Opt. Express 19, 14370 (2011).

[8] S. Strauf and F. Jahnke, Laser Photon. Rev. 5, 607 (2011).

[9] W. W. Chow and F. Jahnke, Prog. Quant. Electron. 37, 109 (2013)

[10] C. Schneider, A. Rahimi-Iman, N. Y. Kim, J. Fischer, I. G. Savenko, M. Amthor, M. Lermer, A. Wolf,
L. Worschech, V. D. Kulakovskii, I. A. Shelykh, M. Kamp, S. Reitzenstein, A. Forchel, Y. Yamamoto, and S. Höfling, Nature 497, 348 (2013)

[11] T. Akiyama, M. Sugawara, and Y. Arakawa, Proc. IEEE 95, 1757 (2007)

[12 P. Michler, A. Kiraz, C. Becher, W. Schoenfeld, P. Petroff, L. Zhang, E. Hu, and A. Imamoglu, Science 290, $2282(2000)$

[13] C. Santori, D. Fattal, and Y. Yamamoto, Single-photon Devices and Applications (Wiley, Weinheim, 2010).

[14] S. Buckley, K. Rivoire, and J. Vučković, Rep. Prog. Phys. 75, 126503 (2012).

15] P. Lodahl, S. Mahmoodian, and S. Stobbe, Rev. Mod. Phys. 87, 347 (2015).

[16] H. J. Kimble, Nature 453, 1023 (2008)

[17] N. Gisin, G. Ribordy, W. Tittel, and H. Zbinden, Rev. Mod. Phys. 74, 145 (2002)

[18] E. Knill, R. Laflamme, and G. J. Milburn, Nature 409, 46 (2001) 
[19] B. A. Kairdolf, A. M. Smith, T. H. Stokes, M. D. Wang, A. N. Young, and S. Nie, Annu. Rev. Anal. Chem. 6, 143 (2013)

[20] A. Steinhoff, P. Gartner, M. Florian, and F. Jahnke, Phys. Rev. B 85, 205144 (2012).

[21] W. van Roosbroeck, Bell Syst. Tech. J. 29, 560 (1950).

[22] A. Martí, L. Cuadra, and A. Luque, IEEE T. Electron Dev. 49, 1632 (2002).

[23] M. Gioannini, A. P. Cedola, N. D. Santo, F. Bertazzi, and F. Cappelluti, IEEE J. Photovolt. 3, 1271 (2013).

[24] M. Kantner, U. Bandelow, T. Koprucki, J.-H. Schulze, A. Strittmatter, and H.-J. Wünsche, IEEE T. Electron Dev. 63, 2036 (2016).

[25] E. B. Davies, Comm. Math. Phys. 39, 91 (1974)

[26] G. Lindblad, Comm. Math. Phys. 48, 119 (1976).

[27] V. Gorini, A. Kossakowski, and E. C. G. Sudarshan, J. Math. Phys. 17, 821 (1976).

[28] H.-P. Breuer and F. Petruccione, The Theory of Open Quantum Systems (Oxford University Press, 2002).

[29] M. Grupen and K. Hess, IEEE J. Quantum Elect. 34, 120 (1998)

[30] S. Steiger, R. G. Veprek, and B. Witzigmann, J. Comput. Electron. 7, 509 (2008)

[31] T. Koprucki, A. Wilms, A. Knorr, and U. Bandelow, Opt. Quant. Electron. 42, 777 (2011)

[32] S. Selberherr, Analysis and Simulation of Semiconductor Devices (Springer, Wien, 1984).

[33] S. R. de Groot and P. Mazur, Non-equilibrium Thermodynamics, Dover Books on Physics (Dover Publications, 1984).

[34] G. Schaller, Open quantum systems far from equilibrium, Lecture Notes in Physics, Vol. 881 (Springer, 2014).

[35] R. Alicki, Rep. Math. Phys. 10, 249 (1976)

[36] A. Kossakowski, A. Frigerio, V. Gorini, and M. Verri, Comm. Math. Phys. 57, 97 (1977)

[37] G. B. Cuetara, M. Esposito, and G. Schaller, Entropy 18, 447 (2016)

[38] J. Gemmer, M. Michel, and G. Mahler, Quantum Thermodynamics, Lecture Notes in Physics, Vol. 657 (Springer, Berlin, Heidelberg, 2004).

[39] R. Kosloff, Entropy 15, 2100 (2013).

[40] M. Esposito, M. A. Ochoa, and M. Galperin, Phys. Rev. Lett. 114, $080602(2015)$

[41] J. Goold, M. Huber, A. Riera, L. del Rio, and P. Skrzypczyk, J. Phys. A 49, 143001 (2016).

[42] J. P. Pekola, Nature Physics 11, 118 (2015)

[43] P. Strasberg, G. Schaller, T. Brandes, and M. Esposito, Phys. Rev. X 7, 021003 (2017).

[44] G. Albinus, H. Gajewski, and R. Hünlich, Nonlinearity 15, 367 (2002)

[45] G. Albinus, ZAMM-Z. Angew. Math Mech. (ICIAM/ GAMM 95 Applied Analysis) 76, 289 (1996).

[46] R. Alicki, Int. J. Theor. Phys. 16, 351 (1977).

[47] I. Magnúsdóttir, A. V. Uskov, S. Bischoff, B. Tromborg, and J. Mørk, J. Appl. Phys. 92, 5982 (2002).

[48] T. R. Nielsen, P. Gartner, and F. Jahnke, Phys. Rev. B 69, $235314(2004)$.

[49] E. Malić, K. J. Ahn, M. J. P. Bormann, P. Hövel, M. Kuntz, D. Bimberg, A. Knorr, and E. Schöll, IEEE J. Sel. Top. Quant. 13, 1242 (2007)

[50] M.-R. Dachner, E. Malić, M. Richter, A. Carmele, J. Kabuss, A. Wilms, J.-E. Kim, G. Hartmann, J. Wolters, U. Bandelow, and A. Knorr, Phys. Status Solidi B 247, 809 (2010).
[51] A. Wilms, P. Mathé, F. Schulze, T. Koprucki, A. Knorr, and U. Bandelow, Phys. Rev. B 88, 235421 (2013).

[52] H. Gajewski and K. Gärtner, ZAMM-Z. Angew. Math Mech. 76, 247 (1996)

[53] H. Spohn, J. Math. Phys. 19, 1227 (1978)

[54] M. Grmela and H. C. Ottinger, Phys. Rev. E 56, 6620 (1997)

[55] H. C. Öttinger, Europhys. Lett. 94, 10006 (2011)

[56] A. Mielke, in Mathematical Results in Quantum Mechanics, edited by P. Exner, W. König, and H. Neidhardt (World Scientific, Singapore, 2015) pp. 331-348.

[57] M. Mittnenzweig and A. Mielke, J. Stat. Phys. 167, 205 (2017)

[58] Z. Yuan, B. E. Kardynal, R. M. Stevenson, A. J. Shields, C. J. Lobo, K. Cooper, N. S. Beattie, D. A. Ritchie, and M. Pepper, Science 295, 102 (2002)

[59] A. J. Bennett, R. B. Patel, A. J. Shields, K. Cooper, P. Atkinson, C. A. Nicoll, and D. A. Ritchie, Appl. Phys. Lett. 92, 193503 (2008)

[60] W. Unrau, D. Quandt, J.-H. Schulze, T. Heindel, T. D. Germann, O. Hitzemann, A. Strittmatter, S. Reitzenstein, U. W. Pohl, and D. Bimberg, Appl. Phys. Lett. 101, 211119 (2012)

[61] A. Schlehahn, R. Schmidt, C. Hopfmann, J.-H. Schulze, A. Strittmatter, T. Heindel, L. Gantz, E. Schmidgall, D. Gershoni, and S. Reitzenstein, Appl. Phys. Lett. 108, 021104 (2016)

[62] A. Wojs, P. Hawrylak, S. Fafard, and L. Jacak, Phys. Rev. B 54, 5604 (1996)

[63] N. Baer, P. Gartner, and F. Jahnke, Eur. Phys. J. B 42, 231 (2004)

[64] V. Weisskopf and E. Wigner, Z. Phys. 63, 54 (1930),

[65] M. Florian, C. Gies, P. Gartner, and F. Jahnke, J. Opt. Soc. Am. B 29, A31 (2012).

[66] R. Ferreira and G. Bastard, Capture and Relaxation in Self-Assembled Semiconductor Quantum Dots (Morgan \& Claypool Publishers, 2015).

[67] P. Farrell, N. Rotundo, D. H. Doan, M. Kantner, J. Fuhrmann, and T. Koprucki, "Handbook of optoelectronic device modeling and simulation," (CRC Press, 2017) Chap. Mathematical Methods: Drift-Diffusion Models, pp. 731-769, to appear.

[68] D. L. Scharfetter and H. K. Gummel, IEEE T. Electron Dev. 16, 64 (1969)

[69] M. Bessemoulin-Chatard, Numer. Math. 121, 637 (2012)

[70] T. Koprucki, N. Rotundo, P. Farrell, D. H. Doan, and J. Fuhrmann, Opt. Quant. Electron. 47, 1327 (2014)

[71] M. Kantner and T. Koprucki, Opt. Quant. Electron. 48, 543 (2016).

[72] S. Selberherr, in Proc. of the 172nd Meeting of the Electrochem. Soc., Vol. 88-9 (Honolulu, 1987) pp. 70-86.

[73] D. M. Richey, J. D. Cressler, and R. C. Jaeger, J. Phys. IV France 04, C6 (1994).

[74] V. Zwiller, T. Aichele, and O. Benson, New J. Phys. 6, 96 (2004)

[75] A. Jüngel, Transport Equations for Semiconductors, Lecture Notes in Physics, Vol. 773 (Springer, Berlin, Heidelberg, 2009).

[76] V. Palankovski and R. Quay, Analysis and Simulation of Heterostructure Devices, Computational Microelectronics (Springer Science \& Business Media, Vienna, 2004).

[77] T. Mnatsakanov, M. Levinshtein, L. Pomortseva, and S. Yurkov, Semiconductors 38, 56 (2004).

[78] N. F. Mott, Rev. Mod. Phys. 40, 677 (1968) 